\title{
Clinical and Neurobiological Aspects of TAO Kinase Family in Neurodevelopmental Disorders
}

\author{
Chun $\mathrm{Hu}^{1,2 * t}$, Pan Feng ${ }^{1,2}$, Qian Yang ${ }^{1,2}$ and Lin Xiao ${ }^{1,2 *}$ \\ ${ }^{1}$ Key Laboratory of Brain, Cognition and Education Sciences, Ministry of Education, South China Normal University, \\ Guangzhou, China, ${ }^{2}$ Institute for Brain Research and Rehabilitation, South China Normal University, Guangzhou, China
}

Despite the complexity of neurodevelopmental disorders (NDDs), from their genotype to phenotype, in the last few decades substantial progress has been made in understanding their pathophysiology. Recent accumulating evidence shows the relevance of genetic variants in thousand and one (TAO) kinases as major contributors to several NDDs. Although it is well-known that TAO kinases are a highly conserved family of STE20 kinase and play important roles in multiple biological processes, the emerging roles of TAO kinases in neurodevelopment and NDDs have yet to be intensively discussed. In this review article, we summarize the potential roles of the TAO

\section{OPEN ACCESS}

Edited by:

Tania Cristina Leite de Sampaio e Spohr,

Instituto Estadual do Cérebro Paulo Niemeyer (IECPN), Brazil

Reviewed by: Froylan Calderon De Anda, University of Hamburg, Germany Benjamin Cogne, Centre Hospitalier Universitaire (CHU) de Nantes, France

*Correspondence: Chun Hu huchun603@163.com Lin Xiao

liuyangxiaolin@aliyun.com

${ }^{\dagger}$ Lead contact

Received: 18 January 2021 Accepted: 04 March 2021 Published: 24 March 2021

Citation: Hu C, Feng $P$, Yang $Q$ and Xiao $L$ (2021) Clinical and Neurobiological Aspects of Tao Kinase Family in Neurodevelopmental Disorders. Front. Mol. Neurosci. 14:655037. doi: 10.3389/fnmol.2021.655037 kinases based on structural and biochemical analyses, present the genetic data from clinical investigations, and assess the mechanistic link between the mutations of TAO kinases, neuropathology, and behavioral impairment in NDDs. We then offer potential perspectives from basic research to clinical therapies, which may contribute to fully understanding how TAO kinases are involved in NDDs.

Keywords: TAO kinase, neurodevelopmental disorders, neuron, cytoskeleton, de novo mutations, therapy

\section{INTRODUCTION}

Neurodevelopmental disorders (NDDs) are a group of heterogeneous conditions that fail to acquire multiple proper developmental milestones involved in cognitive, emotional, and psychomotor skills caused by abnormal changes in early brain development. NDDs include mainly autism spectrum disorder (ASD), intellectual disability (ID), developmental delay (DD), attention deficit hyperactivity disorder (ADHD), schizophrenia, and epilepsy and affect around 3-5\% of children worldwide (Fitzgerald et al., 2015; Wilfert et al., 2017; Parenti et al., 2020; Hanly et al., 2021). In general, any possible factor that could disrupt tightly programmed and coordinated events will eventually result in NDDs (Parenti et al., 2020). Although NDDs have been intensively investigated for many years, from the bench to the clinic, little is known about their causal risk factors and/or genes and fundamental neurobiology due to their complexities from their genotypes to phenotypes (Sullivan and Geschwind, 2019; Moyses-Oliveira et al., 2020; Parenti et al., 2020). One of the most productive areas of NDDs research, however, lies in human genetics. The combined genome-wide association study (GWAS; Visscher et al., 2017) and next generation techniques based on whole genome and exome sequencing (WGS and WES; Sanders et al., 2018; Coe et al., 2019; Satterstrom et al., 2020), have identified quite a few of susceptive genes for developing NDDs, which provides further opportunities to study the underlying mechanisms of how genetic mutations contribute to specific NDDs. Thus, to identify reproducible high risk 
genes and to reveal their underlying neurobiological mechanisms in molecular, cellular, and circuit-levels is critical for developing personalized treatments for NDDs (Krystal and State, 2014).

TAO (Thousand and one) kinase family belongs to the STE20 group kinases and consists of three genes in vertebrates including TAOK1, TAOK2, and TAOK3 that encode TAO1, TAO2, and TAO3, respectively (Dan et al., 2001; Miller et al., 2019). They have relatively close homologs in several invertebrate species like C. elegans and Drosophila, which structurally contain three conserved domains including the kinase domain, central domain and regulatory domain (Figure 1A). TAO kinases were reported to play multifunctional roles in many molecular and cellular events by interacting with MAPK cascade, MST family kinases, the cytoskeleton, and apoptosisassociated proteins (Duan et al., 2020). Therefore, it is not surprisingly that TAO kinases could regulate neuronal survival (Wakabayashi et al., 2005; Wu and Wang, 2008; Li et al., 2019) and development (Yasuda et al., 2007; de Anda et al., 2012; Ultanir et al., 2014; Yadav et al., 2017; Richter et al., 2018; Dulovic-Mahlow et al., 2019) in the nervous system. Although there is plenty of evidence suggesting that TAO kinases likely contribute to NDDs, the direct association between TAO kinases and NDDs has not been fully discovered until recently.

The original discovery of two genomic deletions in TAOK1 is from patients with developmental delay (nsv1062993; Cooper et al., 2011), microcephaly, and seizures (Decipher 250045; Decipher database; Xie et al., 2016). In addition, Xie et al. (2016) also identified a 17q11.2 microdeletion that covers TAOK1 in a patient with developmental delay and postnatal microcephaly, implying the copy number variants (CNVs) of TAOK1 is likely involved in NDDs. Following the exome sequencing of over 4,200 individuals with developmental disorders, researchers found four missense de novo mutations in TAOK1 that likely contribute to those developmental disorders (Deciphering Developmental Disorders Study, 2017). Subsequently, a WES based analysis further identified eight de novo mutations in TAOK1 from eight patients with different NDDs including speech and language development and/or motor development delay, muscular hypotonia, macrocephaly, seizures, and intellectual disability (Dulovic-Mahlow et al., 2019). In addition, a total 23 patients with NDDs who carry different monogenic TAOK1 variants (20 individuals) or a microdeletion covering TAOK1 (three individuals) were discovered in a recent study (Woerden et al., 2021). These studies provide solid evidence that both pathogenic CNVs and single point mutations that disrupt TAOK1 could have a deleterious effect that contributes to NDDs.

TAOK2 is supposed to be a susceptive gene of NDDs and is based on the findings that $16 \mathrm{p} 11.2$ microdeletion or microduplication contributes to multiple NDDs (Weiss et al., 2008; McCarthy et al., 2009; Steinman et al., 2016). Human TAOK2 is one of the 30 genes located in the microdeletion or microduplication region of $16 \mathrm{p} 11.2$. By performing wholegenome sequencing (WGS) of families with ASD, one frame shift deletion of TAOK2 was identified (Yuen et al., 2017). This was further confirmed in a following study (Richter et al., 2018), in which two other more de novo mutations of TAOK2 was identified in ASD patients (Richter et al., 2018).

Compared to those repeatedly identified TAOK1 and TAOK2 de novo mutations in NDDs, less attention was paid to the TAOK3 gene. A previous analysis of de novo CNVs by WGS in individuals suffering from bipolar disorder and schizophrenia showed that a microdeletion that affects TAOK3 (and PEBP1) is present in schizophrenia patient (Malhotra et al., 2011) and TAOK3 (but not PEBP1) was further confirmed in a GWAS analysis (Gilman et al., 2012), suggesting that TAOK3 alone may contribute to NDDs, at least in schizophrenia. The direct evidence for monogenic TAOK3 mutations contributing to NDDs is from a comprehensive study with WES, which systematically analyzed over 2,500 autistic children and 1,900 unaffected siblings and the parents of each family and eventually identified two validated de novo mutations of TAOK3 in ASD patients (Iossifov et al., 2014). It would be beneficial to identify more monogenic mutations of TAOK3 in ASD or other NDDs in future studies.

Because each individual TAO kinase was reported to be associated with NDDs, it is important to investigate and obtain new insights into their biological mechanisms in neurodevelopment to confirm their potential roles in NDDs. Thus, despite the progress made in both basic and clinical research of TAO kinases related NDDs in the last few years, the systematic summary of these findings are lacking. In this review, we will introduce TAO kinases functions in general, summarize the de novo mutations that contribute to multiple NDDs, and discuss the possible underlying molecular and cellular mechanisms. Lastly, we propose future directions of an intensive understanding of TAO kinases in neurodevelopment and NDDs, which might be critical in the development of potential and precise treatment of specific NDDs.

\section{TAO KINASES IN GENERAL}

\section{TAO Kinase Structure and Expression Patterns in the Central Nervous System (CNS)}

The TAO (Thousand and one) kinase subfamily is part of the larger STE20 (sterile 20) kinase family, a diverse group of serine-threonine kinases that participate in a variety of signaling pathways (Dan et al., 2001). The TAO kinase family is highly conserved, and has three members in mammals including TAOK1/PSK2/MARKK (Hutchison et al., 1998), TAOK2/PSK1 (Moore et al., 2000), and TAOK3/JIK (Tassi et al., 1999), but just a single representative TAO kinase in C. elegans (Kin-18; Berman et al., 2001; Spiga et al., 2013; Yin et al., 2016) and Drosophila melanogaster (Tao); (Liu et al., 2010). Structurally, all members of TAO kinases contain three domains including the $\mathrm{N}$-terminal catalytic domain, the central domain, and the C-terminal regulatory domain which regulates catalytic activity (Figure 1A). Among these domains, the catalytic domains of all TAO kinase members are extremely conserved, but the regulatory domains may determine their distinct functions. In addition, each member of 


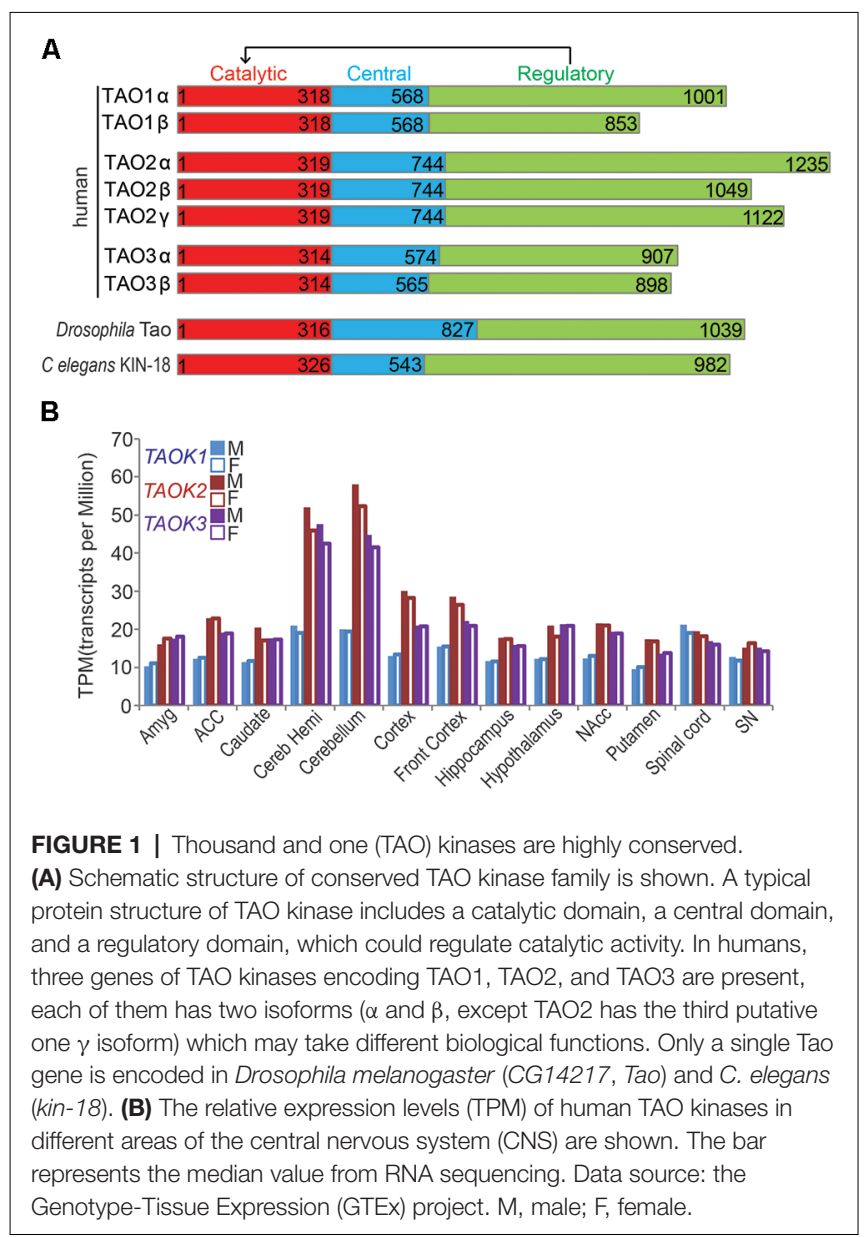

mammalian TAO kinases has two isoforms ( $\alpha$ and $\beta$ ) except for TAO2, which has a putative third one (TAO2 $\gamma$ ) which is mainly distinguished by the regulatory domain (Figure 1A), suggesting a distinct biological function of the different isoforms. Thus, it was reported that the two isoforms ( $\alpha$ and $\beta$ ) of TAO2 play different roles in dendritic and dendritic spine development (Yasuda et al., 2007; Richter et al., 2018), the possible isoform-dependent functions in other members should be further explored.

TAO kinases are ubiquitously expressed without obvious tissue specificity based on mRNA transcription and protein translational levels (Duan et al., 2020), although it was reported that TAOK1 and TAOK2 are highly enriched in the brain by Northern blot detection (Hutchison et al., 1998). In the CNS, TAO kinases are widely distributed in all brain regions and the spinal cord without gender differences ${ }^{1}$ (Figure 1B). In addition, TAO kinases are modestly expressed but TAOK2 and TAOK3 showed strong expression in the cerebellum (including cerebellar hemisphere) and cortex (including front cortex), implying that the importance of these brain regions are critical for TAO kinase related NDDs.

\footnotetext{
${ }^{1}$ https://www.gtexportal.org/
}

\section{The General Functions of TAO Kinases}

As TAO kinases belong to the STE20 kinase family, the original functions of TAO kinase are supposed to act as upstream regulators of mitogen activated protein kinases (MAPKs). Upon extracellular stimulations, conventional MAPK cascades including the ERK1/2, p38 MAPK $\alpha, \beta, \delta$, and $\gamma$, and JNK $1 / 2 / 3$ pathways are selectively activated to integrate, amplify, and regulate signal transduction and eventually affect multiple biological processes including cell proliferation, differentiation, migration, and apoptosis (Johnson and Lapadat, 2002; Kyosseva, 2004; Cargnello and Roux, 2011). TAO1 and TAO2 have a lot in common regarding activating p38 MAPK (Hutchison et al., 1998; Chen et al., 1999) and the JNK cascade (Moore et al., 2000; Zihni et al., 2006). TAO3 shows a relatively distinct function that activates ERKs (Zhang et al., 2000) and p38 MAPK but inhibits the JNK cascade (Tassi et al., 1999). It should be noted that TAO3 may inhibit p38 kinases in certain conditions (Tassi et al., 1999). Thus, the TAO kinases family is a central regulator in controlling MAPKs cascades and may be involved in multiple biological processes (Figure 2).

In addition, TAO1 is also known as MARKK (microtubule affinity regulating kinase) due to its ability to phosphorylate MARK, which can further affect microtubule (MT) arrangement by modulating tau (Giacomini et al., 2018) and other related microtubule-associated proteins (MAPs; Drewes et al., 1997), indicating the functional role of TAO1 in regulating MT dynamics. Further research found that TAO1 could interact with Spred-1 to inhibit TESK1, which could modulate actin dynamics by activating Cofilin (Moore et al., 2000). At the same time, TAO2 was also reported to modulate actin filament and MT rearrangement in cultured 3T3 cells (Moore et al., 2000; Mitsopoulos et al., 2003). Although direct evidence remains is scarce, TAO3 might also play a role in the regulation of cytoskeletal dynamics due to the fact that it has been reported that this kinase modulates the JNK signaling (Kapfhamer et al., 2012; Zeke et al., 2016). It is therefore not surprising that Drosophila Tao, the only homolog of mammalian Tao kinases, affects actin and MT dynamics in cultured Drosophila S2 cells (Liu et al., 2010; Pflanz et al., 2015) and primordial germ cells from developing embryos (Pflanz et al., 2015). It should be noted that the MAPK cascade signaling pathway has a tight connection with cytoskeletal dynamics (Šamaj et al., 2004; Komis et al., 2011). Therefore, TAO kinases' functions may be tightly involved in cytoskeletal dynamics regulation (Figure 2).

\section{CLINICAL ASSOCIATION BETWEEN TAO KINASES AND NDDs}

Because TAO kinases is intensively involved in regulating cytoskeletal dynamics, which is required for nearly all processes of normal neuronal and glia development involving cellular survival, migration, polarity, differentiation and plasticity (Conde and Cáceres, 2009; Hoogenraad and Bradke, 2009; Kapitein and Hoogenraad, 2015; Konietzny et al., 2017; Weigel et al., 2020), they are always speculated to be risk genes for NDDs. However, this reasonable speculation has not, until 


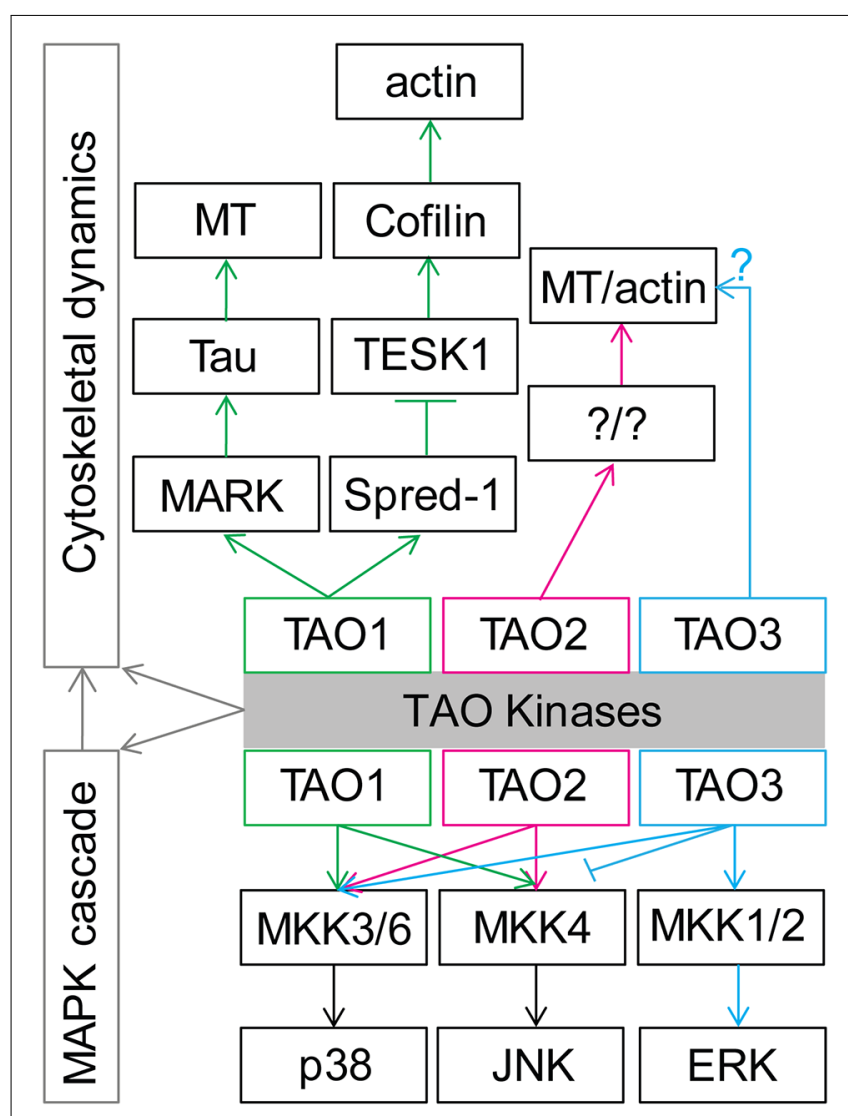

FIGURE 2 | General functions of TAO kinases in regulation of cytoskeletal dynamics and mitogen activated protein kinase (MAPK) cascade cytoskeletal dynamics. See the main text for details. Note that TAO1 and TAO2 share similar functions, although TAO1 preferentially activates p38 and TAO2 preferentially activates JNK. Importantly, the possible distinct function between TAO3 and TAO1/2 should be further examined.

recently, been proven to be correct, benefiting from powerful next- generation sequencing techniques and the establishment of well-organized cohorts [e.g., Simons Simplex Collection (SSC) cohort; Fischbach and Lord, 2010] and networks [e.g., the Deciphering Developmental Disorders (DDD) network; Firth and Wright, 2011] of investigators.

\section{TAOK1}

A rare de novo microdeletion at $17 \mathrm{q} 11.2$, which covers TAOK1, was identified in a patient with a developmental delay and postnatal microcephaly (Xie et al., 2016), suggesting a possible role of TAOK1 to contribute to NDDs. Two genomic microdeletions in the TAOK1 genome (without affecting other genes) in patients with developmental delay (nsv1062993; Cooper et al., 2011), microcephaly, and seizures (Decipher \#250045, Decipher database) was reported. Importantly, the deletion nsv1062993 completely overlaps the CNV of \#250045, and the two partially overlap the TAOK1 gene. It is assumed that the TAOK1 gene plays a pivotal role in the phenotype of patients (Xie et al., 2016). In addition, TAOK1 was predicted to have a haploinsufficiency score less than 10 , suggesting one copy deletion of TAOK1 could cause clinical consequences (Xie et al.,
2016). Followed by an integrated meta-analysis that combines de novo mutations from exome sequencing data and $\mathrm{CNV}$ morbidity data, TAOK1 with a missense mutation was identified as a candidate for NDDs (Coe et al., 2019). Furthermore, in a large scale whole exome screening (WES) of patients with autism, TAOK1 was identified as one of over 100 putative autism spectrum disorder (ASD) associated genes (Satterstrom et al., 2020). In addition, TAOK1 de novo mutations were identified from eight children with associated NDDs including delayed speech and language development (5/8), autism (2/8), intellectual deficiency (4/8), macrocephaly (3/8), and motor development delay (6/8; Dulovic-Mahlow et al., 2019). Interestingly, half of these de novo mutations locate in the catalytic domain, one truncated mutation is present in the central domain, and the other three are truncated or frameshifted mutations in the regulatory domain. Although the precise functional analysis of these mutations is still lacking, the blood and fibroblast line derived from a patient carrying a variant (c.2366_2367insC) was analyzed. It was found that this variant could decrease cDNA (Sanger sequencing), mRNA (quantitative real time PCR), and protein (western blot) levels of TAOK1. In addition, the mutated mRNA could be stabilized by cycloheximide treatment indicating that nonsense-mediated mRNA decay could explain the reduced abundance of the mutant allele (Dulovic-Mahlow et al., 2019). Interestingly, a recent study collected a cohort of 23 individuals with NDDs from a collaboration facilitated by GeneMatcher (Sobreira et al., 2015) with multiple de novo variants in TAOK1, among which 20 individuals had an intragenic TAOK1 variant and three patients had a chromosomal deletion including TAOK1 (Woerden et al., 2021). Importantly, this study tested several variants with functional assays in a mouse model showing that two variants (c.500T $>\mathrm{G}$ and c.943C $>\mathrm{T}$ ) may act with dominant negative $(\mathrm{DN})$ functions and one variant $(\mathrm{c} .1643 \mathrm{~T}>\mathrm{C})$ may have a loss of function (LOF) effect. Thus, these studies provide clear evidence that dysfunction of TAOK1 with either CNVs or mutations with DN or LOF effect will result in NDDs.

\section{TAOK2}

TAOK2 is highlighted as a candidate risk gene for NDDs because previous reports showing that microdeletion and microduplication of the $16 \mathrm{p} 11.2$ genetic locus are associated with ASDs or schizophrenia (Weiss et al., 2008; McCarthy et al., 2009; Zheng et al., 2013). TAOK2 is one of the 30 genes that are located in the 16p11.2 region. Recently, more psychiatric features like speech/language impairments, intellectual disability, motor/developmental delay, microcephaly and macrocephaly have been identified in patients with 16p11.2 microdeletion and microduplication (Steinman et al., 2016; Rein and Yan, 2020). Although it has been long been proposed that disruption of monogenic TAOK2 function might result in NDDs, the clear clinical evidence has only been presented recently. By performing whole-genome sequencing (WGS) of families with ASD, one frame shift deletion of TAOK2 was identified (Yuen et al., 2017). This was further confirmed in a following study (Richter et al., 2018). Richter et al. (2018) combined WGS and WES to examine over 2,600 families with ASDs and identified 24 different variants in TAOK2, including the frame shift deletion identified 
by Yuen et al. (2017). They further characterized that three of these variants are known to be de novo including a missense mutation in the catalytic domain (A135P), a frameshift deletion resulting in truncation $\left(\mathrm{P} 1022^{*}\right)$ in the regulatory domain, and a de novo splice site variant $\left(c .563+12 \_563+15 \mathrm{del}\right)$ predicted to cause intron seven retention. Among these three de novo mutations, A135P mutation results in lower levels of both phosphorylated and total TAO2 but the P1022* mutation does not affect TAO2 levels in patients with derived lymphoblastoid cells. Combined with more biochemical analysis, A135P and P1022* were confirmed to abolish (LOF) and enhance (GOF) kinase activity, respectively. The impact of the de novo splice site variant $\left(c .563+12 \_563+15 \mathrm{del}\right)$, however, is still unknown and requires further characterization (Richter et al., 2018). It should be noted that, patients carrying these de novo mutations showed typical ASDs symptoms and also have a certain degree of disability or delay in language and speech development, implying the broad function of TAOK2 in neurodevelopment.

Although TAOK2 contribution to $16 \mathrm{p} 11.2 \mathrm{CNVs}$ pathophysiology is quite convincing, it should be noted that it is TAOK2 and other genes (e.g., MAPK3, SEZ6L2 and KCTD13) that act collectively to develop much complex and diverse 16p11.2 CNVs phenotypes (Krishnan et al., 2016), compared to individual gene mutations (Richter et al., 2018; Rein and Yan, 2020). In this review, we focus on TAOK2 function only since 16p11.2 CNVs related NDDs have recently been reviewed (Rein and Yan, 2020) and is also out of scope of the current study.

\section{TAOK3}

Unlike TAOK1 and TAOK2, studies on the association of TAOK3 with NDDs is relatively scarce. Several GWAS based studies indicate that TAOK3 is likely involved in NDDs. A study with a rare $\mathrm{CNV}$ s analysis by WGS suggested that a de novo deletion that affects TAOK3 (and PEBP1) may contribute to schizophrenia (Malhotra et al., 2011) and TAOK3 (but not PEBP1) was further confirmed in a GWAS analysis (Gilman et al., 2012), suggesting LOF of monogenic TAOK3 may contribute to NDDs, at least in schizophrenia. In another GWAS study, TAOK3 was identified as a genetic predisposition to loneliness (Abdellaoui et al., 2019), a status that accompanies NDDs (Kwan et al., 2020; Papagavriel et al., 2020). In addition, two independent GWAS analyses showed that TAOK3 is related to high opioid requirement for patients with advanced cancer pain (Gutteridge et al., 2018) and morphine requirement for postoperative pain in a retrospective pediatric day surgery population (CookSather et al., 2014), suggesting a functional role of TAOK3 in controlling pain under certain conditions. Since the impairment of sensory perception/processing is highly associated with NDDs, like autism (Robertson and Baron-Cohen, 2017), the possible function of TAOK3 in NDDs is further implied. Except for GWAS studies, in a genome-wide-association meta-analysis (GWAMA), TAOK3 was also identified as a susceptive gene for depression (Baselmans et al., 2019). Overall, those studies suggest a possible role of TAOK3 in NDDs.

The direct evidence for monogenic TAOK 3 links to NDDs is based on a large scale WES that compared and analyzed over 2,500 affected ASD children and 1,900 unaffected siblings and the parents of each family (Iossifov et al., 2014). In this systematical study, two novel missense de novo mutations TAOK3 (c.1495A $>\mathrm{G}$ and c.1894C $>$ T) were identified and validated (Iossifov et al., 2014; Table 1). These two missense mutations encode protein TAO3 with pT199A and pR632W, which are located in the central and regulatory domain, respectively. Although they are likely deleterious mutations as predicted in the gnomAD database, it should be noted that to determine whether these two mutations in TAOK3 are pathogenic or not requires a detailed functional analysis and more clinical samples. Thus, it would be better elucidated if more deleterious mutations of TAOK3 in ASD or other NDDs patients are identified and validated in future studies.

\section{NEURONAL MECHANISMS OF TAO KINASES IN NEURODEVELOPMENT AND NDDs}

TAO kinases mutations that caused multiple NDDs could be a result of neurological and/or non-neurological impairments. So far, however, the non-neurological roles of TAO kinases in neurodevelopment and NDDs has not been reported. We will focus on the neurological aspect and summarize the accumulated data that implies an essential role of TAO kinases in regulating neuronal development (Figure 3), survival, and maturation.

\section{TAO Kinases Regulate Dendrite Development}

Although TAO kinases at the mRNA level are widely expressed in adult human brains (Figure 1B), TAO2 is preferentially highly expressed in the intermediate zone and the cortical plate of the developing cortex (E18) in mice (de Anda et al., 2012). Interestingly, $\mathrm{TAO} 2 \alpha$ is expressed from early embryonic stages (E10) to adults but $\mathrm{TAO} 2 \beta$ is only detectable at late stage (from E19) in the mouse brain cortex. Immunostaining with mouse cortical neurons (E17) and cultured 2 days in vitro, $\mathrm{TAO} 2$ is found to locate in the growth cone where actin is enriched and its active form pTAO2 was found to localize at the neurite shaft where MT is accumulated (de Anda et al., 2012), suggesting a possible role of TAO2 in regulating neuronal cytoskeletal dynamics. Knockdown of Taok2 decreases basal dendrite arborization and callosal axon projection in the developing cortex (de Anda et al., 2012). Moreover, TAO2 could act downstream of the secreted guidance cue Semaphorin $3 \mathrm{~A}$ (Sema3A) by interacting with its receptor Neuropilin 1 (Nrp1) to activate the JNK signaling pathway (de Anda et al., 2012).

To further characterize the neurodevelopmental functions of Taok2 in mice, Richter et al. (2018) systematically analyze the neurological phenotypes in Taok2 knockout (KO) mice, which were previously found to control behavioral response to ethanol in mice (Kapfhamer et al., 2013). Magnetic resonance imaging (MRI) of fixed 8- to 10-week-old mouse brains of Taok2 KO and $\operatorname{HET}(\mathrm{KO} /+)$ brains found that their absolute brain volumes were significantly enlarged compared with wild type (WT) mice but have a relative decrease in the somatosensory cortex and corpus callosum in a gene dose dependent way. Behavioral 
TABLE 1 | A selected list of de novo mutations between thousand and one (TAO) kinases and neurodevelopmental disorders (NDDs).

\begin{tabular}{|c|c|c|c|c|c|c|}
\hline & Variants & $\begin{array}{l}\text { Amino } \\
\text { acid } \\
\text { change }\end{array}$ & Monogenic & Data type & Diagnosis & Reference/source \\
\hline $\begin{array}{l}\text { TAOK1 } \\
\text { (NM_020791.2) }\end{array}$ & c. $50 A>G$ & $\mathrm{E} 17 \mathrm{G}$ & Yes & WES & $\begin{array}{l}\text { Delayed speech and language; } \\
\text { ID; ADHD }\end{array}$ & $\begin{array}{l}\text { Dulovic-Mahlow et al. } \\
\text { (2019) }\end{array}$ \\
\hline (NM_020791.2) & c. $332 \mathrm{C}>\mathrm{T}$ & S111F & Yes & WES & $\begin{array}{l}\text { Delayed speech and language; } \\
\text { ID; ASD }\end{array}$ & $\begin{array}{l}\text { Dulovic-Mahlow et al. } \\
\text { (2019) }\end{array}$ \\
\hline (NM_020791.2) & c. $892 A>G$ & K298E & Yes & WES & $\begin{array}{l}\text { Delayed speech and language; } \\
\text { Macrocephaly; ASD }\end{array}$ & $\begin{array}{l}\text { Dulovic-Mahlow et al. } \\
\text { (2019) }\end{array}$ \\
\hline (NM_020791.2) & c. $914 \mathrm{~A}>\mathrm{C}$ & D305A & Yes & WES & ID & $\begin{array}{l}\text { Dulovic-Mahlow et al. } \\
\text { (2019) }\end{array}$ \\
\hline (NM_020791.2) & c. $1630 \mathrm{C}>\mathrm{T}$ & Q554* & Yes & WES & ADHD & $\begin{array}{l}\text { Dulovic-Mahlow et al. } \\
\text { (2019) }\end{array}$ \\
\hline (NM_020791.2) & c. $2341 \mathrm{G}>\mathrm{T}$ & $\mathrm{E} 781^{*}$ & Yes & WES & ID; Macrocephaly & $\begin{array}{l}\text { Dulovic-Mahlow et al. } \\
\text { (2019) }\end{array}$ \\
\hline (NM_020791.2) & c.2366_2367insC & L790Ffs*3 & Yes & WES & $\begin{array}{l}\text { Delayed speech and language; } \\
\text { ID; ASD }\end{array}$ & $\begin{array}{l}\text { Dulovic-Mahlow et al. } \\
\text { (2019) }\end{array}$ \\
\hline (NM_020791.2) & c. $2488 \mathrm{G}>\mathrm{T}$ & E830* & Yes & WES & Delayed speech and language & $\begin{array}{l}\text { Dulovic-Mahlow et al. } \\
\text { (2019) }\end{array}$ \\
\hline (NM_020791.2) & c. $656 \mathrm{C}>\mathrm{T}$ & A219V & Yes & WES & Developmental disorders & $\begin{array}{l}\text { Deciphering Developmental } \\
\text { Disorders Study (2017) }\end{array}$ \\
\hline (NM_020791.2) & c.500T>G & L167R & Yes & WES & Developmental disorders & $\begin{array}{l}\text { Deciphering Developmental } \\
\text { Disorders Study (2017) and } \\
\text { Woerden et al. (2021) }\end{array}$ \\
\hline (NM_020791.2) & c. $70 \mathrm{C}>\mathrm{A}$ & P24T & Yes & WES & Developmental disorders & $\begin{array}{l}\text { Deciphering Developmental } \\
\text { Disorders Study (2017) }\end{array}$ \\
\hline (NM_020791.2) & c. $865 \mathrm{G}>\mathrm{A}$ & V289M & Yes & WES & Developmental disorders & $\begin{array}{l}\text { Deciphering Developmental } \\
\text { Disorders Study (2017) }\end{array}$ \\
\hline (NM_020791.2) & c. $943 \mathrm{C}>\mathrm{T}$ & L315F & Yes & WES & NDDs & Woerden et al. (2021) \\
\hline (NM_020791.2) & c. $1643 \mathrm{~T}>\mathrm{C}$ & L548P & Yes & WES & NDDs & Woerden et al. (2021) \\
\hline (NM_020791.2) & $\begin{array}{l}\text { Partial } \\
\text { deletion } \\
\text { chr17:277713 } \\
42-27809321\end{array}$ & $\mathrm{P} ?$ & Yes & WGS & Microcephaly and seizures & $\begin{array}{l}\text { Decipher database } \\
\text { (Xie et al., 2016) }\end{array}$ \\
\hline (NM_020791.2) & $\begin{array}{l}\text { Partial } \\
\text { deletion } \\
\text { chr17:277305 } \\
\text { 73-27802767 }\end{array}$ & $P ?$ & Yes & WGS & Developmental delay & $\begin{array}{l}\text { Cooper et al. (2011) and Xie } \\
\text { et al. (2016) }\end{array}$ \\
\hline $\begin{array}{l}\text { 17q11.2 } \\
\text { (covers TAOK1) }\end{array}$ & Deletion & & No & WGS & $\begin{array}{l}\text { Developmental delay and } \\
\text { postnatal microcephaly }\end{array}$ & Xie et al. (2016) \\
\hline $\begin{array}{l}\text { 17q11.2 } \\
\text { (covers TAOK1) }\end{array}$ & Deletion & & No & WES & NDD & Woerden et al. (2021) \\
\hline $\begin{array}{l}\text { TAOK2 } \\
(\text { NM_016151) }\end{array}$ & c. $403 \mathrm{G}>\mathrm{C}$ & $\mathrm{A} 135 \mathrm{P}$ & Yes & WES, WGS & ASD & Richter et al. (2018) \\
\hline (NM_004783) & c.3057_3081del & P1022* & Yes & WES, WGS & ASD; Delayed language & $\begin{array}{l}\text { Yuen et al. (2017) and } \\
\text { Richter et al. (2018) }\end{array}$ \\
\hline (NC_000016.9) & $\begin{array}{l}\text { c. } 563+12- \\
563+15\end{array}$ & $P ?$ & Yes & WES, WGS & ASD & Richter et al. (2018) \\
\hline $\begin{array}{l}\text { 16p11.2 } \\
\text { (covers TaoK2) }\end{array}$ & Deletion & & No & WGS & ASD & Weiss et al. (2008) \\
\hline $\begin{array}{l}16 \mathrm{p} 11.2 \\
\text { (covers TaoK2) }\end{array}$ & Duplication & & No & WGS & ASD, Schizophrenia & $\begin{array}{l}\text { Weiss et al. (2008) and } \\
\text { McCarthy et al. (2009) }\end{array}$ \\
\hline TAOK3 & Deletion & & No & WGS, GWAS & Schizophrenia & $\begin{array}{l}\text { Malhotra et al. (2011) and } \\
\text { Gilman et al. (2012) }\end{array}$ \\
\hline (NM_016281.3) & c. $1495 A>G$ & T499A & Yes & WES & ASD & lossifov et al. (2014) \\
\hline (NM_016281.3) & c. $1894 \mathrm{C}>\mathrm{T}$ & A632T & Yes & WES & ASD & lossifov et al. (2014) \\
\hline
\end{tabular}

CNVs of 17q11.2 and 16p11.2 locus are shown in the list because they cover TAOK1 and TAOK2, respectively. In total, 20 point mutations of TAOK1 were identified (Woerden et al., 2021) and three of the de novo mutations showing functional changes were selected. Note: the gene with the affected mRNA accession no. is indicated if available in the original studies/database. The individual variant is labeled; deletion or duplication means the whole gene is deleted or duplicated in the genomic fragment while partial deletion means the deletion fragment is located in the affected gene, without affecting other genes. Amino acid change: * indicates the stop codon. WGS, whole genome sequencing; WES, whole exome sequencing; GWAS, genome-wide associated study.

analysis indicated that the impairments on cognition, anxiety, and social interaction in Taok2 $\mathrm{KO}$ mice were consistent with previous clinical studies of ASD patients (Hazlett et al., 2005;
Freitag et al., 2009; Hardan et al., 2009; Vaccarino and Smith, 2009; Lai et al., 2014; Sacco et al., 2015). By examining the dendritic morphology in the prefrontal cortex, they found that 


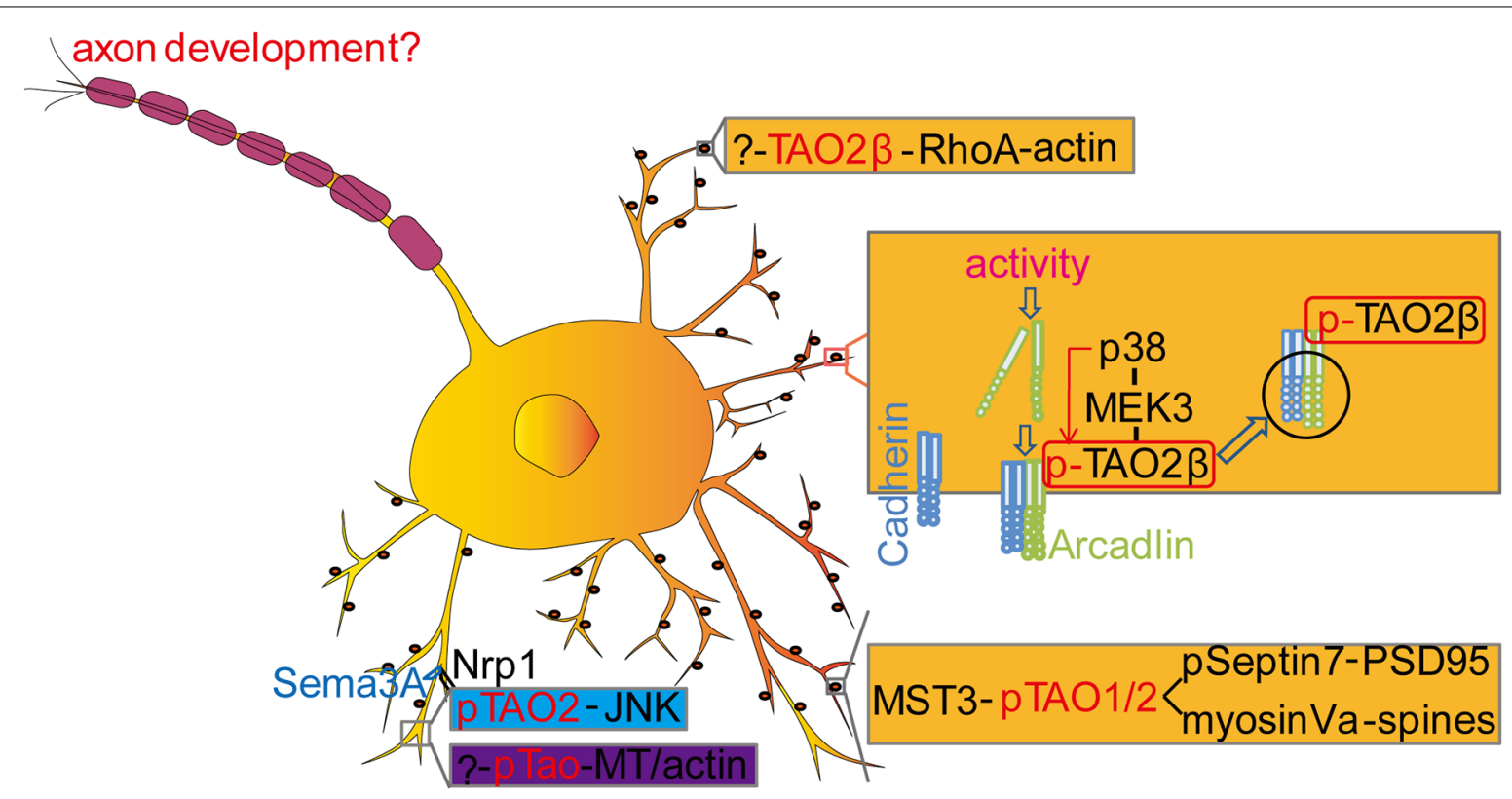

FIGURE 3 | Molecular mechanisms of Tao kinases in neurodevelopment. TAO2 could regulate dendrite development (blue labeled) and spine development (yellow labeled) with multiple signaling pathways. In Drosophila, Tao kinase regulated dendritic development is also indicated with an unknown upstream pathway [?-pTao-Microtubule (MT)/actin] (purple labeled). In addition, TAO2 functions downstream of BDNF to activate the JNK signaling cascade to regulate interneuron maturation during development. However, whether TAO kinases contribute to axonal development and the underlying mechanisms remains to be determined. See details in the main text.

Taok2 KO and HET mice showed a decrease in basal dendritic complexity, which is comparable to the previous in vitro study (de Anda et al., 2012).

A recent study also clearly showed that overexpression (OE) of human TAO1 and its several NDDs linked variants in cultured primary hippocampal neurons from mice, could significantly reduce dendritic arborization and length (Woerden et al., 2021) although the detailed mechanisms remain to be further explored. Thus, to obtain more physiological evidence, further exploration of its function in neuronal/dendrite development, by generating Taok1 KO mice, is required.

We recently also found that Drosophila Tao could significantly affect peripheral dendritic development of dendritic arborization (da) neurons at whole larval developmental stages and in adults ( $\mathrm{Hu}$ et al., 2020). da sensory neurons are a well-characterized model system to study neuronal development and functions (Grueber et al., 2002; Williams and Truman, 2004; Shimono et al., 2009; Jan and Jan, 2010; Im and Galko, 2012; Copf, 2015). Using this model, many of the NDDs related genes and their molecular mechanisms have been confirmed and identified (Gatto and Broadie, 2011; Coll-Tane et al., 2019). We found that Drosophila Tao is expressed in all da sensory neurons and its active form pTao is discretely distributed along dendrites, suggesting a local function of Drosophila Tao in regulation of MT and/or actin to affect dendritic development. Loss of Drosophila Tao increases dendritic complexity and MTs dynamic of all da sensory neurons in vivo in a developmental stage dependent way ( $\mathrm{Hu}$ et al., 2020), which is the opposite to the phenotype of neocortex neurons from an in vitro culture system (de Anda et al., 2012) or TAOK2 KO mice (Richter et al., 2018). Interestingly, this increased dendritic complexity phenotype could be fully rescued by wild type human TAO2 but not by the ASD linked LOF mutation (A135P), suggesting a conserved function of Drosophila Tao and TAO2; but its readout varies in different model systems (King and Heberlein, 2011). Moreover, we also found that disruption of Drosophila Tao in adult sensory neurons caused dendritic over-branching and resulted in impairment of social behaviors, which further confirmed the previous observations that sensory perception is critical for developing ASDs in a mouse model (Orefice et al., 2016, 2019).

Altogether, these different in vitro and in vivo models clearly showed a critical and complex role of TAO kinases in regulating dendrite development which then affects normal brain functions.

\section{TAO Kinases Regulate Spine/Synapse Development}

Both dendrite arborization and synapse formation are critical for wiring the neural circuitry and establishing normal neural functions (Benson et al., 2001; Jan and Jan, 2001; McAllister, 2007; Colón-Ramos, 2009; Batool et al., 2019). Yadav et al. found that $\mathrm{TAO} 2$ localizes to dendritic spines and is required for synaptic maturation in a kinase activity dependent way (Yadav et al., 2017). Combined with an elegant chemical-genetic method and mass spectrometry, the authors identified several candidate substrates of TAO2 including Septin6, Septin7, HADC6, Bail, Caskin1, CEP170, and MST3, and only Septin7 was further confirmed in a subsequent functional analysis. Septin7 is a 
GTP-binding protein (Neubauer and Zieger, 2017) that regulates actin (Hu et al., 2012; Mavrakis et al., 2014) and MT remodeling (Bowen et al., 2011; Hu et al., 2012) to control axon/dendrite branching and spine morphology (Xie et al., 2007; Hu et al., 2012). It was found that TAO2 could directly phosphorylate Septin7 and lead to its trafficking to the dendritic spine where it could associate with and immobilize the synapse scaffolding protein PSD95 to promote spine synapse maturation (Yadav et al., 2017). In addition, a peptide pull-down method to identify binding proteins in neuronal lysates labeled by stable isotope labeling by amino acids in culture (SILAC; Zhang et al., 2011; Deng et al., 2019) was employed to identify possible binding components of TAO1/2, showing that Myosin Va could interact with $\mathrm{TAO} 1 / 2$ in a phosphorylation dependent manner. Moreover, endogenous Myosin $\mathrm{Va}$ could bind endogenous TAO1 and be phosphorylated by TAO1/2 in neurons (Ultanir et al., 2014). Myosin $\mathrm{Va}$ is a motor protein in charge of the intracellular transport of vesicles, organelles, and protein complexes along the actin filaments (Harrington and Rodgers, 1984; Masters et al., 2016; Guhathakurta et al., 2018; Lombardo et al., 2019) and affects microtubule based transport when recruited on the same cargo with the microtubule motor kinesin (Kapitein et al., 2013), suggesting a role of Myosin Va in promoting synaptic formation/maturation when localized in the spine (Ultanir et al., 2014). In addition, the authors found that TAO1/2 could be phosphorylated by mammalian sterile 20 (Ste20)-like kinase 3 (MST3), a homolog to Drosophila Hippo (Harvey et al., 2003). Interestingly, in contrast to the downstream of mammalian Tao Kinase to MST3, it seems Drosophila Tao is an upstream signaling component activating Hippo to regulate tissue growth (Boggiano et al., 2011; Poon et al., 2011, 2018; Huang et al., 2014; Chung et al., 2016). However, whether Drosophila Tao could phosphorylate Hippo to regulate synaptic growth needs to be further explored.

In another study, it was shown that TAO $2 \beta$ instead of TAO $2 \alpha$ is essential for activity-induced dendritic spine formation (Yasuda et al., 2007), which rises a possibility that TAO kinases function in an isoform-dependent way. Electroconvulsive or other excitatory stimuli in cultured hippocampal neurons triggers a protocadherin arcadlin that stimulates TAO2 $\beta$ specifically, which in turn activates p38 MAPK through MEK3, resulting in the endocytosis of $\mathrm{N}$-cadherin and the decrease in spine numbers (Yasuda et al., 2007; Sun and Xie, 2012), suggesting an isoform specific function of TAO kinases in spine development. This finding was further confirmed in the Taok2 KO mouse model (Richter et al., 2018). It was shown that dendritic spines in hippocampal neurons from Taok2 $\mathrm{KO}$ mice were dramatically decreased compared to WT, possibly by directly affecting the RhoA activity, a kinase which is also preferentially combined to TAO $2 \beta$ and is highly involved in the regulation of spine mobility. Those data indicate that the C-terminal of distinct TAO kinases is critical for their unique physiological functions.

Using Drosophila (neuron-muscle junction) NMJ as a classic system for studying synaptic development (Broadie and Bate, 1995; Menon et al., 2013; Frank, 2014) assists neurobiologist in identifying multiple psychiatric disorder related genes and their underlying molecular mechanisms (Sun and Xie, 2012; Tian et al., 2017). Knockdown or deactivation (hypomorphic allele) of Drosophila Tao increases the number of NMJ (buttons, the single NMJ structure; Politano et al., 2019). However, it seems Drosophila Tao that regulates NMJ development is not dependent on the Hippo/MST pathway as it is in hippocampal neurons (Ultanir et al., 2014) but it could negatively regulate BMP signaling as reduction of Drosophila Tao leads to an increase in both nucleic pMad levels and BMP target gene expression in motor neuron (Politano et al., 2019). However, another study recently showed that knockdown of Tao could decrease the NMJ number (Dulovic-Mahlow et al., 2019). Thus, both studies indicated the important role of Drosophila Tao in synaptic development, while the underlying mechanisms for the opposite phenotypes observed independently remains to be illustrated.

We recently developed a novel model for studying synaptic development in Drosophila larvae (Tenedini et al., 2019) to compensate for the NMJ model. At larvae stage, peripheral Class IV da (C4da) sensory neurons directly come into contact with a pair of interneurons A08n that can form a functional synaptic structure (Town et al., 2014; Hu et al., 2017; Kaneko et al., 2017), which is closer to mammalian synapses when compared with the classic NMJ synaptic system. By using the Syp-GRASP technique (Macpherson et al., 2015) to label C4da-A08n synapses, we found that loss of Tao results in exuberant postsynaptic (comparable to spine structure) specializations and aberrant connectivity during larval growth. Using functional imaging and a behavioral analysis we showed that loss of Drosophila Tao could induce ectopic functional synapses formation of the A08n neuron with other types (C3da) of neurons and resulted in altered behavioral responses in a connection-specific manner (Tenedini et al., 2019), indicating that TAO kinase mutations, like other NDDs susceptive genes, can induce abnormal behaviors partially from improper establishment of neural circuits (Kida and Kato, 2015; Kaiser et al., 2017; Südhof, 2017).

\section{TAO Kinases Control Neuronal Apoptosis and Maturation via the JNK Signaling Cascade}

The association between neuronal apoptosis and NDDs is not well documented. A previous study showed that overexpression (OE) of the full length or kinase domain of human TAO1 in human neuroblastoma SH-SY5Y cells resulted in cellular apoptosis which was indicated by elevated caspase-3 activity. However, OE of the regulatory domain of TAO1 in SH-SY5Y did not appear to have an obvious change ( $\mathrm{Wu}$ and Wang, 2008). Importantly, OE of TAO1 induced elevated caspase-3like activity and apoptosis of neuroblastoma could be reduced by JNK inhibitor SP600125 to some extent. In addition, OE of rat TAO3 in PC12 (a widely used cell line with the properties of intersecting neurons) resulted in elevation of the expression level of BimEL (Wakabayashi et al., 2005), a protein with apoptotic activity. Recently, it was also reported that TAO1 protected MCAO-induced cerebral ischemic stroke by decreasing the pro-inflammatory factors and apoptosis via PI3K/AKT and 
MAPK signaling pathways (Li et al., 2019), which further indicates the critical roles of TAO kinases in neuronal apoptosis. Those preliminary studies suggest an underestimated function of TAO kinases in neuronal apoptosis.

Furthermore, TAO2 may play roles in the regulation of neuronal maturation. A recent study showed that BDNF could regulate cortical GABAergic interneuron maturation in a TAO2-JNK signaling pathway in a 16p11.2 duplication mouse model (Willis et al., 2020). Cultured neurons from 16p11.2 duplication mice exhibit an abnormal interneuron developmental phenotype that may be involved in a premature closure of the critical period which is likely to be driven by $\mathrm{OE}$ of TAO2 and the subsequent over-activity of JNK, since pharmacological inhibition of TAO kinase could alleviate the $16 \mathrm{p} 11.2$ duplication phenotype. Given the importance of parvalbumin $(\mathrm{PBV}+)$ interneurons in the regulation of excitatory/inhibitory balance within cortical regions, accelerated GABAergic development by TAO2 over-activity may lead to dysregulated network activity and synaptic connectivity (Willis et al., 2020). In addition, it was reported that OE of human TAO1 could also prevent maturation of cultured primary hippocampal neurons from mice (Woerden et al., 2021), while the role of JNKs is involvement is yet to be determined.

\section{TAO Kinases Regulate Neuronal Migration}

A recent study investigated a possible function of TAOK1 in neuronal migration, a process that is critical for normal brain development (Woerden et al., 2021). By employing the in utero electroporation in mice at embryonic day 14.5 (a well-established time window when immature neurons generated from progenitor cells start to migrate to their final destination within the cortical plate, which will eventually form the cerebral cortex layer 2/3), the authors found that knockdown of Taok1 resulted in a clear migration deficit of the neurons when compared to control neurons from postnatal day 1 (p1) to P7, when only $75 \%$ of the Taok 1 knockdown neurons were present in cerebral cortex layer $2 / 3$, compared to $95 \%$ in control conditions (Woerden et al., 2021). Similar migration deficits were observed when transfection of several NDDs, linked human TAOK1 variants in developing mouse brains including c.500T $>\mathrm{G}$ and c. $943 \mathrm{C}>\mathrm{T}$ (see Table 1), suggesting a possible role of TAOK1 in neuronal migration during early human brain development.

\section{CONCLUSION AND PERSPECTIVE}

There is solid evidence showing that all TAO kinase members are involved in NDDs including ASD, schizophrenia, and language/speech development delays, implying an indispensable function of the TAO kinase family in neurodevelopment. Although several animal models from Drosophila to knockout mice provides us with a preliminary role of TAO kinases in neuronal development, survival and maturation, the detailed mechanisms of how TAO kinases contribute to NDDs still need to be investigated.

First, the neurobiological functions of TAO kinases should be further investigated. At the molecular level, identification of specific up- and down-stream substrates for TAO kinases in different model systems, especially by combining human induced pluripotent stem cell (iPSC) from patients, is crucial for a better understanding of the mechanisms leading to the onset of a disease-phenotype (Parenti et al., 2020; Zhang et al., 2020). At cellular level, previous reports focus on the cell-autonomous role of TAO kinases in regulating the development of dendrites and dendritic spines. Whether cell-non-autonomous (e.g., different types of glia cells) roles of TAO kinases in neuronal development and functions are present is not reported. In addition, TAO2 was identified to affect axonal development (de Anda et al., 2012). Whether the mechanisms of TAO kinases controlling dendrite/spine development is distinct from axonal development remains unknown. To confirm and further characterize the TAO kinases functions in neurodevelopment or NDDs, non-human primate models are a promising direction.

Second, an obvious question on how Tao kinases contribute to establish and/or modulate disease-related circuits remains unanswered. In Taok2 knockout mice, the oscillatory events were similar in the PFC and slightly decreased in the HC compared to WT littermates. However, the duration, amplitude, and power in theta $(4-7,12 \mathrm{~Hz})$, beta $(12-30 \mathrm{~Hz})$, gamma $(30-100 \mathrm{~Hz})$ frequency ranges and coherence within the beta band were significantly increased, suggesting alterations in $\mathrm{HC}$ and PFC connectivity (Richter et al., 2018).The limitation of this study is that the animals are studied in an anesthetized status, which may affect the actual physiological responses (Chini et al., 2019). In addition, whether restoring the oscillation pattern by optogenetic stimulation could rescue related phenotypes is also an interesting question that is worth further investigation. A recent study showed a disruption of hippocampal-orbitofrontalamygdala connectivity in $16 \mathrm{p} 11.2$ duplication mice. Whether and how much Taok2 contributes to this defect needs to be investigated (Bristow et al., 2020).

In addition, the possible non-neurological functions of TAO kinases in developing NDDs should also be highlighted. Increasing evidence suggests a non-neurological role in NDDs including immunity, gut, and microbiota (Cryan and Dinan, 2012; Sharon et al., 2016; Dinan and Cryan, 2017; Stefano et al., 2018; Fattorusso et al., 2019; Pape et al., 2019). Studies investigating the brain-gut axis demonstrate a critical role for the gut microbiota in orchestrating brain development and behavior, and the immune system is emerging as an important regulator of these interactions. Accordingly, both the gut microbiota and immune system are implicated in the etiopathogenesis or manifestation of NDDs (Fung et al., 2017). TAO kinases were reported to regulate immunity (Ormonde et al., 2018, 2019; Zhang et al., 2018), gut development (Huang et al., 2014) and likely in maintenance of microbiota integration since MAPKs are involved (Thomas and Versalovic, 2010). Thus, the possible non-neurological role of TAO kinases in NDDs should be seriously considered. TAO kinases are ubiquitously expressed in all tissues including in the immune and gastrointestinal system (Duan et al., 2020). However, it is still unknown whether TAO kinases play any role in the regulation of the immune system and the coordination of brain-gut axis functions that affects neurodevelopment and contributes to NDDs. 


\section{without treatment Developing potential therapies for TAO kinases mutations caused NDDs with treatment}

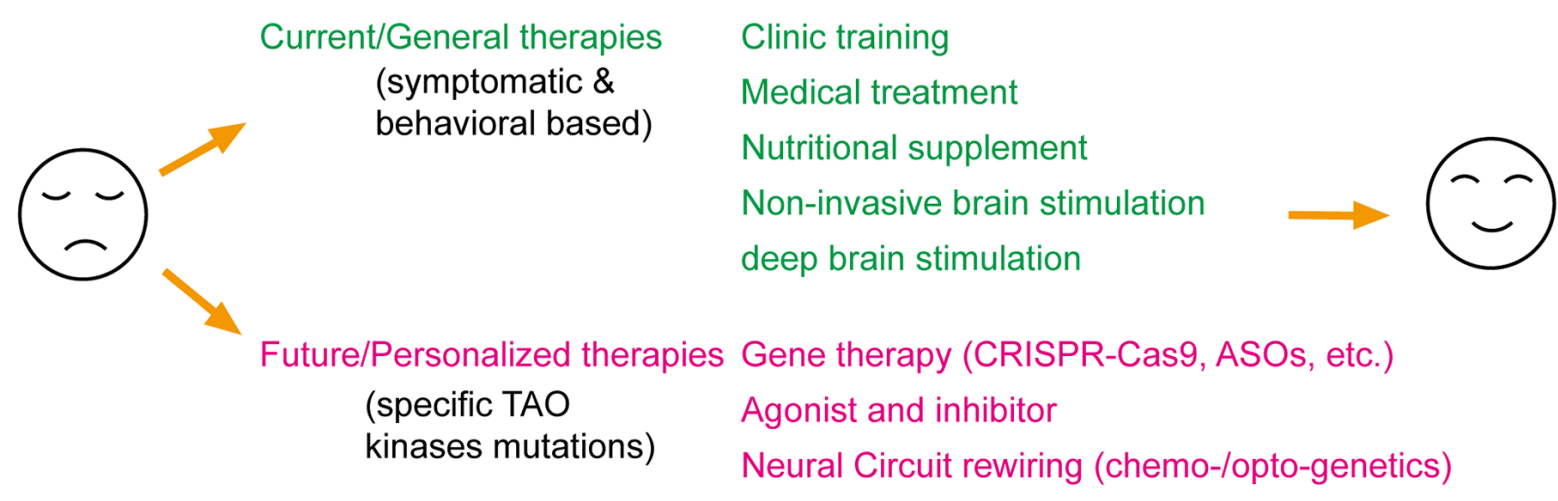

FIGURE 4 | Perspectives on developing potential therapies for TAO kinases mutations caused neurodevelopmental disorders (NDDs). Current therapies include most symptomatic and behavioral based treatments which could be applied to all factors caused NDDs including traditional clinical training, medical treatment, nutritional supplementation, and brain stimulations. With significant progress in biotechnology, the concept for future therapies could focus on personalized therapy by specifically targeting TAO kinase mutations by gene therapy, developing specific agonist or inhibitors and rewiring neural circuits with chemo- or optogenetics.

The eventual goal of investigating the roles and functions of TAO kinases in NDDs is to develop potential therapeutic approaches (Figure 4). Although several above-mentioned basic research discoveries may provide directions for developing potential therapies, clinical trails or even pre-clinical studies on treatment of TAO kinases related NDDs are still lacking. Current clinical training intervention and medical treatments offered for NDDs are symptomatic and behavioral based therapy (Levy and Barak, 2021), which could be applied to any possible factors caused by NDDs. In addition, nutritional supplements (Chang and Su, 2020), non-invasive brain stimulation [including transcranial direct current stimulation (tDCS) and transcranial magnetic stimulation (TMS)] (Khaleghi et al., 2020), and deep brain stimulation in certain cases (Beszej et al., 2019; Lin et al., 2019) are being or will be applied in clinical treatments of NDDs.

Although those general treatments could rescue certain symptoms and behaviors, developing precision medicine that directly and specifically targets disease-causing mutations would be more beneficial (Javed et al., 2020). To functionally rescue TAO kinases mutations caused NDDs, the principle of a long-term rebalancing of their kinase activity should always be followed. One possible strategy is to develop agonists and inhibitors with high specificity and potency for TAO kinases (Fang et al., 2020; Ye et al., 2020). Similar to all standardized drug development processes, the efficacy and toxicity in animal models should be cautiously evaluated before these candidates are applied in human clinical trials. In addition, with the significant progress of gene editing and manipulation in the last decade, gene therapy techniques are a promising direction for personalized medical treatment including CRISPR-Cas9 gene-editing for mutation replacement and antisense oligonucleotides (ASOs) for modifying protein levels (Levy and Barak, 2021). It should be noted that most of those gene therapies are still in preclinical testing stages and the number of successful clinical trials is quite limited because it is faced with critical issues when implemented in in vivo, such as limited efficiency, off-target effects, time window and delivery methods, all suggesting that much more effort is required to improve the bench-to-bedside success rate (Levy and Barak, 2021). Theoretically, all those strategies could also be applied to the specific down-stream targets (like Septin7, myosin Va, JNK and RhoA) of TAO kinases which requires further exploration of the signaling network of TAO kinases.

Alternatively, another option is bypassing molecular deficits and directly treating the neural circuit level deficits (Javed et al., 2020). It is widely accepted that NDDs arise from the alteration of normal brain developmental trajectories disrupting the function of specific neural circuits caused by genetic mutations (del Pino et al., 2018). To rewire the neural circuits by chemoand optogenetic manipulations is a promising strategy to treat NDDs (Ferguson and Gao, 2018). The successful application of optogenetic tools to rebuild the functional neural circuit from dorsal raphe to nucleus accumbens that rescues the social defects in 16p11.2 del mice (Walsh et al., 2018) may prove therapeutically beneficial. Thus, the specific neural circuit defects in TAO kinases related NDDs requires further exploration.

In short, human genetic studies and animal models have linked all TAO kinase members to NDDs such as ASD and schizophrenia. The underlying mechanisms of how TAO kinases regulate neurodevelopment, and how their mutations contribute to NDDs are only just emerging. To dissect more detailed and precise mechanisms is a prerequisite in developing personalized therapies for TAO kinases mutations caused NDDs.

\section{AUTHOR CONTRIBUTIONS}

$\mathrm{CH}$ wrote the manuscript, prepared the figures, and finalized the manuscript. LX contributed to writing and commenting. PF 
and QY contributed to figure and table preparation. All authors contributed to the article and approved the submitted version.

\section{FUNDING}

This work was supported by grants from the National Natural Science Foundation of China (32000690 and 31970913), Key-Area Research and Development Program of

\section{REFERENCES}

Abdellaoui, A., Sanchez-Roige, S., Sealock, J., Treur, J. L., Dennis, J., Fontanillas, P., et al. (2019). Phenome-wide investigation of health outcomes associated with genetic predisposition to loneliness. Hum. Mol. Genet. 28, 3853-3865. doi: $10.1093 / \mathrm{hmg} / \mathrm{ddz} 219$

Baselmans, B. M. L., Jansen, R., Ip, H. F., van Dongen, J., Abdellaoui, A., van de Weijer, M. P., et al. (2019). Multivariate genome-wide analyses of the well-being spectrum. Nat. Genet. 51, 445-451. doi: 10.1038/s41588-018-0320-8

Batool, S., Raza, H., Zaidi, J., Riaz, S., Hasan, S., and Syed, N. I. (2019). Synapse formation: from cellular and molecular mechanisms to neurodevelopmental and neurodegenerative disorders. J. Neurophysiol. 121, 1381-1397. doi: 10.1152/jn.00833.2018

Benson, D. L., Colman, D. R., and Huntley, G. W. (2001). Molecules, maps and synapse specificity. Nat. Rev. Neurosci. 2, 899-909. doi: 10.1038/351 04078

Berman, K. S., Hutchison, M., Avery, L., and Cobb, M. H. (2001). kin18, a C. elegans protein kinase involved in feeding. Gene 279, 137-147. doi: 10.1016/s0378-1119(01)00752-1

Beszej, J. A., Wieczorek, T., Kobyko, A., Piotrowski, P., Siwicki, D., Weiser, A., et al. (2019). Deep brain stimulation: new possibilities for the treatment of mental disorders. Psychiatr. Pol. 53, 789-806. doi: 10.12740/PP/OnlineFirst/ 103090

Boggiano, J. C., Vanderzalm, P. J., and Fehon, R. G. (2011). Tao-1 phosphorylates Hippo/MST kinases to regulate the Hippo-Salvador-Warts tumor suppressor pathway. Dev. Cell 21, 888-895. doi: 10.1016/j.devcel.2011.08.028

Bowen, J. R., Hwang, D., Bai, X., Roy, D., and Spiliotis, E. T. (2011). Septin GTPases spatially guide microtubule organization and plus end dynamics in polarizing epithelia. J. Cell Biol. 194, 187-197. doi: 10.1083/jcb.201102076

Bristow, G. C., Thomson, D. M., Openshaw, R. L., Mitchell, E. J., Pratt, J. A., Dawson, N., et al. (2020). 16p11 duplication disrupts hippocampalorbitofrontal-amygdala connectivity, revealing a neural circuit endophenotype for schizophrenia. Cell Rep. 31:107536. doi: 10.1016/j.celrep.2020.107536

Broadie, K., and Bate, M. (1995). The Drosophila NMJ: a genetic model system for synapse formation and function. Semin. Dev. Biol. 6, 221-231. doi: 10.1016/S1044-5781(06)80031-9

Cargnello, M., and Roux, P. P. (2011). Activation and function of the MAPKs and their substrates, the MAPK-activated protein kinases. Microbiol. Mol. Biol. Rev. 75, 50-83. doi: 10.1128/MMBR.00031-10

Chang, J. P. C., and Su, K. P. (2020). Nutritional neuroscience as mainstream of psychiatry: the evidence-based treatment guidelines for using omega-3 fatty acids as a new treatment for psychiatric disorders in children and adolescents. Clin. Psychopharmacol. Neurosci. 18, 469-483. doi: 10.9758/cpn.2020. 18.4.469

Chen, Z., Hutchison, M., and Cobb, M. H. (1999). Isolation of the protein kinase $\mathrm{TAO} 2$ and identification of its mitogen-activated protein kinase/extracellular signal-regulated kinase kinase binding domain. J. Biol. Chem. 274, 28803-28807. doi: 10.1074/jbc.274.40.28803

Chini, M., Gretenkord, S., Kostka, J. K., Pöpplau, J. A., Cornelissen, L., Berde, C. B., et al. (2019). Neural correlates of anesthesia in newborn mice and humans. Front. Neural Circuits 13, 1-13. doi: 10.3389/fncir.2019.00038

Chung, H. L., Augustine, G. J., and Choi, K. W. (2016). Drosophila schip1 links expanded and tao-1 to regulate hippo signaling. Dev. Cell 36, 511-524. doi: 10.1016/j.devcel.2016.02.004

Coe, B. P., Stessman, H. A. F., Sulovari, A., Geisheker, M. R., Bakken, T. E., Lake, A. M., et al. (2019). Neurodevelopmental disease genes implicated by de
Guangdong Province (2019B030335001 and 2018B030332001), and CAS Key Laboratory of Brain Connectome and Manipulation (2019DP173024).

\section{ACKNOWLEDGMENTS}

We would like to apologize to our colleagues whose work was not cited here.

novo mutation and copy number variation morbidity. Nat. Genet. 51, 106-116. doi: 10.1038/s41588-018-0288-4

Coll-Tane, M., Krebbers, A., Castells-Nobau, A., Zweier, C., and Schenck, A. (2019). Intellectual disability and autism spectrum disorders "on the fly”: insights from Drosophila. Dis. Models. Mech. 12:dmm039180. doi: $10.1242 / \mathrm{dmm} .039180$

Colón-Ramos, D. A. (2009). Chapter 2-synapse formation in developing neural circuits. Curr. Top. Dev. Biol. 87, 53-79. doi: 10.1016/S0070-2153(09) 01202-2

Conde, C., and Cáceres, A. (2009). Microtubule assembly, organization and dynamics in axons and dendrites. Nat. Rev. Neurosci. 10, 319-332. doi: $10.1038 / \mathrm{nrn} 2631$

Cook-Sather, S. D., Li, J., Goebel, T. K., Sussman, E. M., Rehman, M. A. and Hakonarson, H. (2014). TAOK3, a novel genome-wide association study locus associated with morphine requirement and postoperative pain in a retrospective pediatric day surgery population. Pain 155, 1773-1783. doi: 10.1016/j.pain.2014.05.032

Cooper, G. M., Coe, B. P., Girirajan, S., Rosenfeld, J. A., Vu, T. H., Baker, C., et al. (2011). A copy number variation morbidity map of developmental delay. Nat. Genet. 43, 838-846. doi: 10.1038/ng.909

Copf, T. (2015). Importance of gene dosage in controlling dendritic arbor formation during development. Eur. J. Neurosci. 42, 2234-2249. doi: 10.1111/ejn.13002

Cryan, J. F., and Dinan, T. G. (2012). Mind-altering microorganisms: the impact of the gut microbiota on brain and behaviour. Nat. Rev. Neurosci. 13, 701-712. doi: $10.1038 / \mathrm{nrn} 3346$

Dan, I., Watanabe, N. M., and Kusumi, A. (2001). The Ste20 group kinases as regulators of MAP kinase cascades. Trends Cell Biol. 11, 220-230. doi: 10.1016/s0962-8924(01)01980-8

de Anda, F. C., Rosario, A. L., Durak, O., Tran, T., Gräff, J., Meletis, K., et al. (2012) Autism spectrum disorder susceptibility gene TAOK2 affects basal dendrite formation in the neocortex. Nat. Neurosci. 15, 1022-1031. doi: 10.1038/ nn.3141

Deciphering Developmental Disorders Study (2017). Prevalence and architecture of de novo mutations in developmental disorders. Nature 542, 433-438. doi: $10.1038 /$ nature21062

del Pino, I., Rico, B., and Marín, O. (2018). Neural circuit dysfunction in mouse models of neurodevelopmental disorders. Curr. Opin. Neurobiol. 48, 174-182. doi: 10.1016/j.conb.2017.12.013

Deng, J., Erdjument-Bromage, H., and Neubert, T. A. (2019). Quantitative comparison of proteomes using SILAC. Curr. Protoc. Protein Sci. 95, 1-14. doi: $10.1002 /$ cpps.74

Dinan, T. G., and Cryan, J. F. (2017). Brain-gut-microbiota axis and mental health. Psychosom. Med. 79, 920-926. doi: 10.1097/PSY.0000000000000519

Drewes, G., Ebneth, A., Preuss, U., Mandelkow, E. M., and Mandelkow, E. (1997). MARK, a novel family of protein kinases that phosphorylate microtubuleassociated proteins and trigger microtubule disruption. Cell 89, 297-308. doi: 10.1016/s0092-8674(00)80208-1

Duan, Q., Ye, J., Shi, M., Chen, W., and Zhu, F. (2020). Research advances in the molecular functions and relevant diseases of TAOKs, novel STE20 kinase family members. Curr. Pharm. Des. 26, 1-12. doi: $10.2174 / 1381612826666200203115458$

Dulovic-Mahlow, M., Trinh, J., Kandaswamy, K. K., Braathen, G. J., Di Donato, N., Rahikkala, E., et al. (2019). De novo variants in TAOK1 cause neurodevelopmental disorders. Am. J. Hum. Genet. 105, 213-220. doi: 10.1016/j.ajhg.2019.05.005 
Fang, C. Y., Lai, T. C., Hsiao, M., and Chang, Y. C. (2020). The diverse roles of tao kinases in health and diseases. Int. J. Mol. Sci. 21, 1-21. doi: 10.3390/ijms21207463

Fattorusso, A., Di Genova, L., Dell'isola, G. B., Mencaroni, E., and Esposito, S. (2019). Autism spectrum disorders and the gut microbiota. Nutrients 11:521. doi: 10.3390/nu11030521

Ferguson, B. R., and Gao, W. J. (2018). Pv interneurons: critical regulators of $\mathrm{E} / \mathrm{I}$ balance for prefrontal cortex-dependent behavior and psychiatric disorders. Front. Neural Circuits 12, 1-13. doi: 10.3389/fncir.2018. 00037

Firth, H. V., and Wright, C. F. (2011). The deciphering developmental disorders (DDD) study. Dev. Med. Child Neurol. 53, 702-703. doi: 10.1111/j.1469-8749. 2011.04032.x

Fischbach, G. D., and Lord, C. (2010). The simons simplex collection: a resource for identification of autism genetic risk factors. Neuron 68, 192-195. doi: 10.1016/j.neuron.2010.10.006

Fitzgerald, T. W., Gerety, S. S., Jones, W. D., Van Kogelenberg, M., King, D. A., McRae, J., et al. (2015). Large-scale discovery of novel genetic causes of developmental disorders. Nature 519, 223-228. doi: 10.1038/nature 14135

Frank, C. A. (2014). Homeostatic plasticity at the Drosophila neuromuscular junction. Neuropharmacology 78, 63-74. doi: 10.1016/j.neuropharm.2013. 06.015

Freitag, C. M., Luders, E., Hulst, H. E., Narr, K. L., Thompson, P. M., Toga, A. W., et al. (2009). Total brain volume and corpus callosum size in medication-naïve adolescents and young adults with autism spectrum disorder. Biol. Psychiatry 66, 316-319. doi: 10.1016/j.biopsych.2009.03.011

Fung, T. C., Olson, C. A., and Hsiao, E. Y. (2017). Interactions between the microbiota, immune and nervous systems in health and disease. Nat. Neurosci. 20, 145-155. doi: 10.1038/nn.4476

Gatto, C. L., and Broadie, K. (2011). Drosophila modeling of heritable neurodevelopmental disorders. Curr. Opin. Neurobiol. 21, 834-841. doi: 10.1016/j.conb.2011.04.009

Giacomini, C., Koo, C.-Y., Yankova, N., Tavares, I. A., Wray, S., Noble, W., et al. (2018). A new TAO kinase inhibitor reduces tau phosphorylation at sites associated with neurodegeneration in human tauopathies. Acta Neuropathol. Commun. 6:37. doi: 10.1186/s40478-018-0539-8

Gilman, S. R., Chang, J., Xu, B., Bawa, T. S., Gogos, J. A., Karayiorgou, M., et al. (2012). Diverse types of genetic variation converge on functional gene networks involved in schizophrenia. Nat. Neurosci. 15, 1723-1728. doi: 10.1038/ nn. 3261

Grueber, W. B., Jan, L. Y., and Jan, Y. N. (2002). Tiling of the Drosophila epidermis by multidendritic sensory neurons. Development 129, 2867-2878.

Guhathakurta, P., Prochniewicz, E., and Thomas, D. D. (2018). Actin-myosin interaction: Structure, function and drug discovery. Int. J. Mol. Sci. 19:2628. doi: 10.3390/ijms19092628

Gutteridge, T., Kumaran, M., Ghosh, S., Fainsinger, R., Klepstad, P., Tarumi, Y., et al. (2018). Single-nucleotide polymorphisms in TAOK3 are associated with high opioid requirement for pain management in patients with advanced cancer admitted to a tertiary palliative care unit. J. Pain Symptom Manage. 56, 560-566. doi: 10.1016/j.jpainsymman.2018.07.011

Hanly, C., Shah, H., Au, P. Y. B., and Murias, K. (2021). Description of neurodevelopmental phenotypes associated with 10 genetic neurodevelopmental disorders: a scoping review. Clin. Genet. 99, 335-346. doi: $10.1111 /$ cge. 13882

Hardan, A. Y., Libove, R. A., Keshavan, M. S., Melhem, N. M., and Minshew, N. J. (2009). A preliminary longitudinal magnetic resonance imaging study of brain volume and cortical thickness in autism. Biol. Psychiatry 66, 320-326. doi: 10.1016/j.biopsych.2009.04.024

Harrington, W. F., and Rodgers, M. E. (1984). MYOSIN. Annu. Rev. Biochem. 53, 35-73. doi: 10.1146/annurev.bi.53.070184.000343

Harvey, K. F., Pfleger, C. M., and Hariharan, I. K. (2003). The Drosophila Mst ortholog, hippo, restricts growth and cell proliferation and promotes apoptosis. Cell 114, 457-467. doi: 10.1016/s0092-8674(03)00557-9

Hazlett, H. C., Poe, M., Gerig, G., Smith, R. G., Provenzale, J., Ross, A., et al. (2005). Magnetic resonance imaging and head circumference study of brain size in autism: birth through age 2 years. Arch. Gen. Psychiatry 62, 1366-1376. doi: 10.1001/archpsyc.62.12.1366
Hoogenraad, C. C., and Bradke, F. (2009). Control of neuronal polarity and plasticity-a renaissance for microtubules? Trends Cell Biol. 19, 669-676. doi: 10.1016/j.tcb.2009.08.006

Hu, C., Kanellopoulos, A. K., Richter, M., Petersen, M., Konietzny, A., Tenedini, F. M., et al. (2020). Conserved tao kinase activity regulates dendritic arborization, cytoskeletal dynamics and sensory function in Drosophila. J. Neurosci. 40, 1819-1833. doi: 10.1523/JNEUROSCI.1846-19.2020

Hu, C., Petersen, M., Hoyer, N., Spitzweck, B., Tenedini, F., Wang, D., et al. (2017). Sensory integration and neuromodulatory feedback facilitate Drosophila mechanonociceptive behavior. Nat. Neurosci. 20, 1085-1095. doi: 10.1038/ nn. 4580

Hu, J., Bai, X., Bowen, J. R., Dolat, L., Korobova, F., Yu, W., et al. (2012). Septin-driven coordination of actin and microtubule remodeling regulates the collateral branching of axons. Curr. Biol. 22, 1109-1115. doi: 10.1016/j.cub. 2012.04.019

Huang, X., Shi, L., Cao, J., He, F., Li, R., Zhang, Y., et al. (2014). The Sterile 20-Like kinase tao controls tissue homeostasis by regulating the hippo pathway in drosophila adult midgut. J. Genet. Genomics 41, 429-438. doi: 10.1016/j.jgg. 2014.05.007

Hutchison, M., Berman, K. S., and Cobb, M. H. (1998). Isolation of TAO1, a protein kinase that activates MEKs in stress- activated protein kinase cascades. J. Biol. Chem. 273, 28625-28632. doi: 10.1074/jbc.273.44. 28625

Im, S. H., and Galko, M. J. (2012). Pokes, sunburn and hot sauce: Drosophila as an emerging model for the biology of nociception. Dev. Dyn. 241, 16-26. doi: $10.1002 /$ dvdy.22737

Iossifov, I., O’Roak, B. J., Sanders, S. J., Ronemus, M., Krumm, N., Levy, D., et al. (2014). The contribution of de novo coding mutations to autism spectrum disorder. Nature 515, 216-221. doi: 10.1038/nature13908

Jan, Y. N., and Jan, L. Y. (2001). Dendrites. Genes Dev. 15, 2627-2641. doi: $10.1101 /$ gad.916501

Jan, Y.-N., and Jan, L. Y. (2010). Branching out: mechanisms of dendritic arborization. Nat. Rev. Neurosci. 11, 316-328. doi: 10.1038/nrn2836

Javed, S., Selliah, T., Lee, Y. J., and Huang, W. H. (2020). Dosage-sensitive genes in autism spectrum disorders: From neurobiology to therapy. Neurosci. Biobehav. Rev. 118, 538-567. doi: 10.1016/j.neubiorev.2020.08.009

Johnson, G. L., and Lapadat, R. (2002). Mitogen-activated protein kinase pathways mediated by ERK, JNK and p38 protein kinases. Science 298, 1911-1912. doi: $10.1126 /$ science. 1072682

Kaiser, T., Zhou, Y., and Feng, G. (2017). Animal models for neuropsychiatric disorders: prospects for circuit intervention. Curr. Opin. Neurobiol. 45, 59-65. doi: 10.1016/j.conb.2017.03.010

Kaneko, T., Macara, A. M., Li, R., Hu, Y., Iwasaki, K., Dunnings, Z., et al. (2017). Correction: serotonergic modulation enables pathway-specific plasticity in a developing sensory circuit in drosophila. Neuron 95:722. doi: 10.1016/j.neuron. 2017.06.034

Kapfhamer, D., King, I., Zou, M. E., Lim, J. P., Heberlein, U., and Wolf, F. W. (2012). JNK pathway activation is controlled by Tao/TAOK3 to modulate ethanol sensitivity. PLoS One 7:e50594. doi: 10.1371/journal.pone.00 50594

Kapfhamer, D., Taylor, S., Zou, M. E., Lim, J. P., Kharazia, V., and Heberlein, U. (2013). Taok2 controls behavioral response to ethanol in mice. Genes Brain Behav. 12, 87-97. doi: 10.1111/j.1601-183X.2012.00834.x

Kapitein, L. C., and Hoogenraad, C. C. (2015). building the neuronal microtubule cytoskeleton. Neuron 87, 492-506. doi: 10.1016/j.neuron.2015. 05.046

Kapitein, L. C., van Bergeijk, P., Lipka, J., Keijzer, N., Wulf, P. S., Katrukha, E. A., et al. (2013). Myosin-V opposes microtubule-based cargo transport and drives directional motility on cortical actin. Curr. Biol. 23, 828-834. doi: 10.1016/j. cub.2013.03.068

Khaleghi, A., Zarafshan, H., Vand, S. R., and Mohammadi, M. R. (2020). Effects of non-invasive neurostimulation on autism spectrum disorder: a systematic review. Clin. Psychopharmacol. Neurosci. 18, 527-552. doi: 10.9758/cpn.2020. 18.4.527

Kida, S., and Kato, T. (2015). Microendophenotypes of psychiatric disorders: phenotypes of psychiatric disorders at the level of molecular dynamics, synapses, neurons and neural circuits. Curr. Mol. Med. 15, 111-118. doi: $10.2174 / 1566524015666150303002128$ 
King, I., and Heberlein, U. (2011). Tao kinases as coordinators of actin and microtubule dynamics in developing neurons. Commun. Integr. Biol. 4, 554-556. doi: 10.4161/cib.4.5.16051

Komis, G., Illés, P., Beck, M., and Šamaj, J. (2011). Microtubules and mitogenactivated protein kinase signalling. Curr. Opin. Plant Biol. 14, 650-657. doi: 10.1016/j.pbi.2011.07.008

Konietzny, A., Bär, J., and Mikhaylova, M. (2017). Dendritic actin cytoskeleton: structure, functions and regulations. Front. Cell. Neurosci. 11:147. doi: 10.3389/fncel.2017.00147

Krishnan, A., Zhang, R., Yao, V., Theesfeld, C. L., Wong, A. K., Tadych, A., et al. (2016). Genome-wide prediction and functional characterization of the genetic basis of autism spectrum disorder. Nat. Neurosci. 19, 1454-1462. doi: 10.1038/nn.4353

Krystal, J. H., and State, M. W. (2014). Psychiatric disorders: diagnosis to therapy. Cell 157, 201-214. doi: 10.1016/j.cell.2014.02.042

Kwan, C., Gitimoghaddam, M., and Collet, J. P. (2020). Effects of social isolation and loneliness in children with neurodevelopmental disabilities: a scoping review. Brain Sci. 10, 1-36. doi: 10.3390/brainsci10110786

Kyosseva, S. V. (2004). Mitogen-activated protein kinase signaling. Int. Rev. Neurobiol. 59, 201-220. doi: 10.1016/S0074-7742(04)59008-6

Lai, M. C., Lombardo, M. V., and Baron-Cohen, S. (2014). Autism. Lancet 383, 896-910. doi: 10.1016/S0140-6736(13)61539-1

Levy, G., and Barak, B. (2021). Postnatal therapeutic approaches in genetic neurodevelopmental disorders. Neural Regen. Res. 16, 414-422. doi: $10.4103 / 1673-5374.293133$

Li, J., Liu, Z., Wang, L., Xu, H., and Wang, Y. (2019). Thousand one kinase 1 protects MCAO-induced cerebral ischemic stroke in rats by decreasing apoptosis and pro-inflammatory factors. Biosci. Rep. 39, 1-11. doi: 10.1042/BSR20190749

Lin, T. C., Lo, Y. C., Lin, H. C., Li, S. J., Lin, S. H., Wu, H. F., et al. (2019). MR imaging central thalamic deep brain stimulation restored autistic-like social deficits in the rat. Brain Stimul. 12, 1410-1420. doi: 10.1016/j.brs.2019. 07.004

Liu, T., Rohn, J. L., Picone, R., Kunda, P., and Baum, B. (2010). Tao-1 is a negative regulator of microtubule plus-end growth. J. Cell Sci. 123, 2708-2716. doi: $10.1242 /$ jcs.068726

Lombardo, A. T., Nelson, S. R., Kennedy, G. G., Trybus, K. M., Walcott, S., and Warshaw, D. M. (2019). Myosin Va transport of liposomes in threedimensional actin networks is modulated by actin filament density, position and polarity. Proc. Natl. Acad. Sci. U S A 116, 8326-8335. doi: 10.1073/pnas. 1901176116

Macpherson, L. J., Zaharieva, E. E., Kearney, P. J., Alpert, M. H., Lin, T. Y., Turan, Z., et al. (2015). Dynamic labeling of neural connections in multiple colours by trans-synaptic fluorescence complementation. Nat. Commun. 6:10024. doi: 10.1038/ncomms 10024

Malhotra, D., McCarthy, S., Michaelson, J. J., Vacic, V., Burdick, K. E., Yoon, S., et al. (2011). High frequencies of de novo cnvs in bipolar disorder and schizophrenia. Neuron 72, 951-963. doi: 10.1016/j.neuron.2011.11.007

Masters, T. A., Kendrick-Jones, J., and Buss, F. (2016). "Myosins: domain organisation, motor properties, physiological roles and cellular functions," in The Actin Cytoskeleton. Handbook of Experimental Pharmacology, vol 235, ed B. Jockusch (Cham: Springer), 77-122. doi: 10.1007/164_2016_29

Mavrakis, M., Azou-Gros, Y., Tsai, F. C., Alvarado, J., Bertin, A., Iv, F., et al. (2014). Septins promote F-actin ring formation by crosslinking actin filaments into curved bundles. Nat. Cell Biol. 16, 322-334. doi: 10.1038/ncb2921

McAllister, A. K. (2007). Dynamic aspects of cns synapse formation. Annu. Rev. Neurosci. 30, 425-450. doi: 10.1146/annurev.neuro.29.051605. 112830

McCarthy, S. E., Makarov, V., Kirov, G., Addington, A. M., McClellan, J., Yoon, S., et al. (2009). Microduplications of 16p11.2 are associated with schizophrenia. Nat. Genet. 41, 1223-1227. doi: 10.1038/ng.474

Menon, K. P., Carrillo, R. A., and Zinn, K. (2013). Development and plasticity of the Drosophila larval neuromuscular junction. Wiley Interdiscip. Rev. Dev. Biol. 2, 647-670. doi: 10.1002/wdev.108

Miller, C. J., Lou, H. J., Simpson, C., Van De Kooij, B., Hak Ha, B., Fisher, O. S., et al. (2019). Comprehensive profiling of the STE20 kinase family defines features essential for selective substrate targeting and signaling output. PLoS One 17:e2006540. doi: 10.1371/journal.pbio.2006540
Mitsopoulos, C., Zihni, C., Garg, R., Ridley, A. J., and Morris, J. D. H. (2003). The prostate-derived sterile 20-like kinase (PSK) regulates microtubule organization and stability. J. Biol. Chem. 278, 18085-18091. doi: 10.1074/jbc. M213064200

Moore, T. M., Garg, R., Johnson, C., Coptcoat, M. J., Ridley, A. J., and Morris, J. D. (2000). PSK, a novel STE20-like kinase derived from prostatic carcinoma that activates the c-Jun N-terminal kinase mitogen-activated protein kinase pathway and regulates actin cytoskeletal organization. J. Biol. Chem. 275, 4311-4322. doi: 10.1074/jbc.275.6.4311

Moyses-Oliveira, M., Yadav, R., Erdin, S., and Talkowski, M. E. (2020). New gene discoveries highlight functional convergence in autism and related neurodevelopmental disorders. Curr. Opin. Genet. Dev. 65, 195-206. doi: 10.1016/j.gde.2020.07.001

Neubauer, K., and Zieger, B. (2017). The mammalian septin interactome. Front. Cell Dev. Biol. 5, 1-9. doi: 10.3389/fcell.2017.00003

Orefice, L. L., Mosko, J. R., Morency, D. T., Wells, M. F., Tasnim, A., Mozeika, S. M., et al. (2019). Targeting peripheral somatosensory neurons to improve tactile-related phenotypes in ASD models. Cell 178, 867-886.e24.doi: 10.1016/j.cell.2019.07.024

Orefice, L. L., Zimmerman, A. L., Chirila, A. M., Sleboda, S. J., Head, J. P., and Ginty, D. D. (2016). Peripheral mechanosensory neuron dysfunction underlies tactile and behavioral deficits in mouse models of ASDs. Cell 166, 299-313. doi: 10.1016/j.cell.2016.05.033

Ormonde, J. V. S., Li, Z., Stegen, C., and Madrenas, J. (2018). TAOK3 regulates canonical TCR signaling by preventing early SHP-1-mediated inactivation of LCK. J. Immunol. 201, 3431-3442. doi: 10.4049/jimmunol.1800284

Ormonde, J. V. S., Nie, Y., and Madrenas, J. (2019). TAOK3, a regulator of LCK-SHP-1 crosstalk during TCR signaling. Crit. Rev. Immunol. 39, 59-81. doi: 10.1615/CritRevImmunol.2019030480

Papagavriel, K., Jones, R., Sheehan, R., Hassiotis, A., and Ali, A. (2020). The association between loneliness and common mental disorders in adults with borderline intellectual impairment. J. Affect. Disord. 277, 954-961. doi: 10.1016/j.jad.2020.09.005

Pape, K., Tamouza, R., Leboyer, M., and Zipp, F. (2019). Immunoneuropsychiatry-novel perspectives on brain disorders. Nat. Rev. Neurol. 15, 317-328. doi: 10.1038/s41582-019-0174-4

Parenti, I., Rabaneda, L. G., Schoen, H., and Novarino, G. (2020). Neurodevelopmental disorders: from genetics to functional pathways. Trends Neurosci. 43, 608-621. doi: 10.1016/j.tins.2020.05.004

Pflanz, R., Voigt, A., Yakulov, T., and Jäckle, H. (2015). Drosophila gene tao-1 encodes proteins with and without a Ste20 kinase domain that affect cytoskeletal architecture and cell migration differently. Open Biol. 5:140161. doi: 10.1098/rsob.140161

Politano, S. F., Salemme, R. R., Ashley, J., López-Rivera, J. A., Bakula, T. A., Puhalla, K. A., et al. (2019). Tao negatively regulates BMP signaling during neuromuscular junction development in drosophila. Dev. Neurobiol. 79, 335-349. doi: 10.1002/dneu.22681

Poon, C. L. C., Lin, J. I., Zhang, X., and Harvey, K. F. (2011). The sterile 20-like kinase Tao-1 controls tissue growth by regulating the salvador-warts-hippo pathway. Dev. Cell 21, 896-906. doi: 10.1016/j.devcel.2011.09.012

Poon, C. L. C., Liu, W., Song, Y., Gomez, M., Kulaberoglu, Y., Zhang, X., et al. (2018). A hippo-like signaling pathway controls tracheal morphogenesis in Drosophila melanogaster. Dev. Cell 47, 564-575. doi: 10.1016/j.devcel.2018. 09.024

Rein, B., and Yan, Z. (2020). 16p11.2 copy number variations and neurodevelopmental disorders. Trends Neurosci. 43, 886-901. doi: 10.1016/j. tins.2020.09.001

Richter, M., Murtaza, N., Scharrenberg, R., White, S. H., Johanns, O., Walker, S., et al. (2018). Altered TAOK2 activity causes autismrelated neurodevelopmental and cognitive abnormalities through RhoA signaling. Mol. Psychiatry 24, 1329-1350. doi: 10.1038/s41380-0180025-5

Robertson, C. E., and Baron-Cohen, S. (2017). Sensory perception in autism. Nat. Rev. Neurosci. 18, 671-684. doi: 10.1038/nrn.2017.112

Sacco, R., Gabriele, S., and Persico, A. M. (2015). Head circumference and brain size in autism spectrum disorder: a systematic review and meta-analysis. Psychiatry Res. 234, 239-251. doi: 10.1016/j.pscychresns.2015. 08.016 
Šamaj, J., Baluška, F., and Hirt, H. (2004). From signal to cell polarity: mitogenactivated protein kinases as sensors and effectors of cytoskeleton dynamicity. J. Exp. Bot. 55, 189-198. doi: 10.1093/jxb/erh012

Sanders, S. J., Neale, B. M., Huang, H., Werling, D. M., An, J. Y., Dong, S., et al. (2018). Publisher correction: whole genome sequencing in psychiatric disorders: the WGSPD consortium. Nat. Neurosci. 21:1017. doi: 10.1038/s41593-018-0102-8

Satterstrom, F. K., Kosmicki, J. A., Wang, J., Breen, M. S., De Rubeis, S., An, J. Y., et al. (2020). Large-scale exome sequencing study implicates both developmental and functional changes in the neurobiology of autism. Cell 180, 568-584. doi: 10.1016/j.cell.2019.12.036

Sharon, G., Sampson, T. R., Geschwind, D. H., and Mazmanian, S. K. (2016). The central nervous system and the gut microbiome. Cell 167, 915-932. doi: 10.1016/j.cell.2016.10.027

Shimono, K., Fujimoto, A., Tsuyama, T., Yamamoto-Kochi, M., Sato, M., Hattori, Y., et al. (2009). Multidendritic sensory neurons in the adult Drosophila abdomen: origins, dendritic morphology and segment- and age-dependent programmed cell death. Neural Dev. 4:37. doi: 10.1186/17498104-4-37

Sobreira, N., Valle, D., and Hamosh, A. (2015). GeneMatcher: a matching tool for connecting investigators with an interest in the same gene. Hum. Mutat. 36, 928-930. doi: 10.1002/humu.22844

Spiga, F. M., Prouteau, M., and Gotta, M. (2013). The TAO kinase KIN-18 regulates contractility and establishment of polarity in the C. elegans embryo. Dev. Biol. 373, 26-38. doi: 10.1016/j.ydbio.2012.10.001

Stefano, G. B., Pilonis, N., Ptacek, R., Raboch, J., Vnukova, M., and Kream, R. M. (2018). Gut, microbiome and brain regulatory axis: relevance to neurodegenerative and psychiatric disorders. Cell. Mol. Neurobiol. 38, 1197-1206. doi: 10.1007/s10571-018-0589-2

Steinman, K. J., Spence, S. J., Ramocki, M. B., Proud, M. B., Kessler, S. K., Marco, E. J., et al. (2016). 16p11.2 deletion and duplication: characterizing neurologic phenotypes in a large clinically ascertained cohort. Am. J. Med. Genet. Part A 170, 2943-2955. doi: 10.1002/ajmg.a.37820

Südhof, T. C. (2017). Synaptic neurexin complexes: a molecular code for the logic of neural circuits. Cell 171, 745-769. doi: 10.1016/j.cell.2017. 10.024

Sullivan, P. F., and Geschwind, D. H. (2019). Defining the genetic, genomic, cellular and diagnostic architectures of psychiatric disorders. Cell 177, 162-183. doi: 10.1016/j.cell.2019.01.015

Sun, M. K., and Xie, W. (2012). Cell adhesion molecules in Drosophila synapse development and function. Sci. China Life Sci. 55, 20-26. doi: 10.1007/s11427012-4273-3

Tassi, E., Biesova, Z., Di Fiore, P. P., Gutkind, J. S., and Wong, W. T. (1999). Human JIK, a novel member of the STE20 kinase family that inhibits JNK and is negatively regulated by epidermal growth factor. J. Biol. Chem. 274, 33287-33295. doi: 10.1074/jbc.274.47.33287

Tenedini, F. M., Sáez González, M., Hu, C., Pedersen, L. H., Petruzzi, M. M., Spitzweck, B., et al. (2019). Maintenance of cell type-specific connectivity and circuit function requires tao kinase. Nat. Commun. 10:3506. doi: 10.1038/s41467-019-11408-1

Thomas, C. M., and Versalovic, J. (2010). Probiotics-host communication modulation of signaling pathways in the intestine. Gut Microbes 1, 1-16. doi: 10.4161/gmic.1.3.11712

Tian, Y., Zhang, Z. C., and Han, J. (2017). Drosophila studies on autism spectrum disorders. Neurosci. Bull. 33, 737-746. doi: 10.1007/s12264-0170166-6

Town, C., Polytechnic, V., Common, W., Bag, P., Cruces, L., Way, W., et al. (2014). Discovery of brainwide neural-behavioral. Science 344, 386-392. doi: $10.1126 /$ science. 1250298

Ultanir, S. K., Yadav, S., Hertz, N. T., Oses-Prieto, J. A., Claxton, S., Burlingame, A. L., et al. (2014). MST3 kinase phosphorylates TAO1/2 to enable Myosin va function in promoting spine synapse development. Neuron 84, 968-982. doi: 10.1016/j.neuron.2014.10.025

Vaccarino, F. M., and Smith, K. M. (2009). Increased brain size in autism-what it will take to solve a mystery. Biol. Psychiatry 66, 313-315. doi: 10.1016/j. biopsych.2009.06.013

Visscher, P. M., Wray, N. R., Zhang, Q., Sklar, P., McCarthy, M. I., Brown, M. A., et al. (2017). 10 years of GWAS discovery: biology, function and translation. Am. J. Hum. Genet. 101, 5-22. doi: 10.1016/j.ajhg.2017. 06.005

Wakabayashi, T., Kosaka, J., and Oshika, T. (2005). JNK inhibitory kinase is up-regulated in retinal ganglion cells after axotomy and enhances BimEL expression level in neuronal cells. J. Neurochem. 95, 526-536. doi: 10.1111/j. 1471-4159.2005.03389.x

Walsh, J. J., Christoffel, D. J., Heifets, B. D., Ben-Dor, G. A., Selimbeyoglu, A., Hung, L. W., et al. (2018). 5-HT release in nucleus accumbens rescues social deficits in mouse autism model. Nature 560, 589-594. doi: 10.1038/s41586-0180416-4

Weigel, M., Wang, L., and Fu, M. M. (2020). Microtubule organization and dynamics in oligodendrocytes, astrocytes and microglia. Dev. Neurobiol. 1-11. doi: $10.1002 /$ dneu.22753

Weiss, L. A., Shen, Y., Korn, J. M., Arking, D. E., Miller, D. T., Fossdal, R., et al. (2008). Association between microdeletion and microduplication at 16p11.2 and autism. N. Engl. J. Med. 358, 667-675. doi: 10.1056/NEJMoa075974

Wilfert, A. B., Sulovari, A., Turner, T. N., Coe, B. P., and Eichler, E. E. (2017). Recurrent de novo mutations in neurodevelopmental disorders: properties and clinical implications. Genome Med. 9:101. doi: 10.1186/s13073-0170498-x

Williams, D. W., and Truman, J. W. (2004). Mechanisms of dendritic elaboration of sensory neurons in Drosophila: insights from in vivo time lapse. J. Neurosci. 24, 1541-1550. doi: 10.1523/JNEUROSCI.4521-03.2004

Willis, A., Pratt, J. A., and Morris, B. J. (2020). BDNF and JNK signaling modulate cortical interneuron and perineuronal net development: implications for schizophrenia-linked 16p11.2 duplication syndrome. Schizophr. Bull. doi: 10.1093/schbul/sbaa139. [Online ahead of print].

Woerden, G. M., Bos, M., Konink, C., Distel, B., Trezza, R. A., Shur, N. E., et al. (2021). TAOK1 is associated with neurodevelopmental disorder and essential for neuronal maturation and cortical development. Hum. Mutat. doi: 10.1002/humu.24176. [Online ahead of print].

$\mathrm{Wu}, \mathrm{M}$., and Wang, S. (2008). Human TAO kinase 1 induces apoptosis in SH-SY5Y cells. Cell Biol. Int. 32, 151-156. doi: 10.1016/j.cellbi.2007.08.006

Xie, B., Fan, X., Lei, Y., Chen, R., Wang, J., Fu, C., et al. (2016). A novel de novo microdeletion at 17q11.2 adjacent to NF1 gene associated with developmental delay, short stature, microcephaly and dysmorphic features. Mol. Cytogenet. 9 , 9-13. doi: 10.1186/s13039-016-0251-y

Xie, Y., Vessey, J. P., Konecna, A., Dahm, R., Macchi, P., and Kiebler, M. A. (2007). The GTP-binding protein septin 7 is critical for dendrite branching and dendritic-spine morphology. Curr. Biol. 17, 1746-1751. doi: 10.1016/j.cub. 2007.08.042

Yadav, S., Oses-Prieto, J. A., Peters, C. J., Zhou, J., Pleasure, S. J., Burlingame, A. L., et al. (2017). TAOK2 kinase mediates PSD95 stability and dendritic Spine maturation through septin7 phosphorylation. Neuron 93, 379-393. doi: 10.1016/j.neuron.2016.12.006

Yasuda, S., Tanaka, H., Sugiura, H., Okamura, K., Sakaguchi, T., Tran, U., et al. (2007). Activity-induced protocadherin arcadlin regulates dendritic spine number by triggering $\mathrm{N}$-cadherin endocytosis via TAO2 $\beta$ and p38 MAP kinases. Neuron 56, 456-471. doi: 10.1016/j.neuron.2007.08.020

Ye, J., Shi, M., Chen, W., Zhu, F., and Duan, Q. (2020). Research advances in the molecular functions and relevant diseases of TAOKs, novel STE20 kinase family members. Curr. Pharm. Des. 26, 3122-3133. doi: $10.2174 / 1381612826666200203115458$

Yin, Y., Donlevy, S., and Smolikove, S. (2016). Coordination of recombination with meiotic progression in the Caenorhabditis elegans germline by KIN-18, a TAO Kinase that regulates the timing of MPK-1 signaling. Genetics 202, 45-59. doi: 10.1534/genetics.115.177295

Yuen, R. K. C., Merico, D., Bookman, M., Howe, J. L., Thiruvahindrapuram, B., Patel, R. V., et al. (2017). Whole genome sequencing resource identifies 18 new candidate genes for autism spectrum disorder. Nat. Neurosci. 20, 602-611. doi: $10.1038 / \mathrm{nn} .4524$

Zeke, A., Misheva, M., Reményi, A., and Bogoyevitch, M. A. (2016). JNK signaling: regulation and functions based on complex protein-protein partnerships. Microbiol. Mol. Biol. Rev. 80, 793-835. doi: 10.1128/MMBR. 00043-14

Zhang, W., Chen, T., Wan, T., He, L., Li, N., Yuan, Z., et al. (2000). Cloning of DPK, a novel dendritic cell-derived protein kinase activating the 
ERK1/ERK2 and JNK/SAPK pathways. Biochem. Biophys. Res. Commun. 274, 872-879. doi: 10.1006/bbrc.2000.3244

Zhang, G., Deinhardt, K., Chao, M. V., and Neubert, T. A. (2011). Study of neurotrophin-3 signaling in primary cultured neurons using multiplex stable isotope labeling with amino acids in cell culture. J. Proteome Res. 10, 2546-2554. doi: 10.1021/pr200016n

Zhang, X., Li, Z., Liu, Y., and Gai, Z. (2020). Great expectations: induced pluripotent stem cell technologies in neurodevelopmental impairments. Int. J. Med. Sci. 18, 459-473. doi: 10.7150/ijms.51842

Zhang, Z., Tang, Z., Ma, X., Sun, K., Fan, L., Fang, J., et al. (2018). TAOK1 negatively regulates IL-17-mediated signaling and inflammation. Cell. Mol. Immunol. 15, 794-802. doi: 10.1038/cmi.2017.158

Zheng, X., Bei, J. X., Xu, H., Lee, J., Chong, S. A., Sim, K., et al. (2013). The association between rare large duplication of 16p11.2 and schizophrenia in the Singaporean Chinese population. Schizophr. Res. 146, 368-369. doi: 10.1016/j. schres.2013.02.029
Zihni, C., Mitsopoulos, C., Tavares, I. A., Ridley, A. J., and Morris, J. D. H. (2006). Prostate-derived sterile 20-like kinase 2 (PSK2) regulates apoptotic morphology via C-Jun N-terminal kinase and Rho kinase-1. J. Biol. Chem. 281, 7317-7323. doi: 10.1074/jbc.M513769200

Conflict of Interest: The authors declare that the research was conducted in the absence of any commercial or financial relationships that could be construed as a potential conflict of interest.

Copyright (c) $2021 \mathrm{Hu}$, Feng, Yang and Xiao. This is an open-access article distributed under the terms of the Creative Commons Attribution License (CC BY). The use, distribution or reproduction in other forums is permitted, provided the original author(s) and the copyright owner(s) are credited and that the original publication in this journal is cited, in accordance with accepted academic practice. No use, distribution or reproduction is permitted which does not comply with these terms. 